\title{
LE CONTRÔLE DE LA GESTION LOCALE PAR LES CHAMBRES RÉGIONALES ET TERRITORIALES DES COMPTES
}

\author{
UDK: $352 / 354$ (44) \\ Izvorni znanstveni rad \\ Primljeno: 15. IX. 2015.
}

\begin{abstract}
Toute décentralisation implique un contrôle de la gestion locale, pour en assurer le caractère démocratique, la régularité juridique et l'efficacité économique. Mais la gestion locale ne se ramène pas uniquement à l'application de règles de droit et ne se confond pas non plus avec une approche strictement économique. C'est pourquoi la mission de la juridiction financière est diverse et spécifique. Elle associe un contrôle juridictionnel sur les comptes locaux et un contrôle de type non juridictionnel sur le respect des règles budgétaires et sur l'efficacité de la dépense publique en examinant la performance du management local. La vérification de la légalité et de la régularité des opérations financières et la mission générale d'évaluation de la gestion, combinent les logiques juridique, économique et managériale, au service de la démocratie locale et d'une décentralisation maîtrisée et transparente.
\end{abstract}

\section{Mots- clés: Chambres régionales des comptes, décentralisation, finances publiques, comptables publics, contrôle budgétaire, gestion, responsabilité, régularité, performance, management, démocratie locale}

La gestion locale a connu d'importantes transformations dans l'histoire. Jusqu'au XXème siècle, les collectivités locales assuraient surtout des tâches administratives et leurs budgets étaient souvent modestes. Le secteur public durant la période libérale classique n'intervenait qu'au strict minimum dans la vie économique et sociale.

$\mathrm{Au}$ lendemain de la première guerre mondiale, les collectivités locales ont dû pallier les défaillances du secteur privé, se charger de la reconstruction notamment en matière de logement, remédier aux pénuries alimentaires, dans un cadre juridique délimité par la loi et la jurisprudence ${ }^{1}$. Ce rôle s'est ensuite accru en raison de la crise des années 1929-1930. Après la seconde guerre mondiale, les acteurs locaux sont devenus à la fois des administrateurs et des bâtisseurs, sans disposer d'une pleine autonomie toutefois, car l'Etat exerçait un contrôle

1 Le Conseil d'Etat soumettait l'interventionnisme communal à certaines conditions, dont la recherche de l'intérêt général ou l'existence de circonstances locales particulières. Voir A. KOUEVI, Le droit des interventions économiques des collectivités locales, L.G.D.J., 2003. 
administratif étroit et déterminait les politiques locales par l'intermédiaire des préfets.

Les transformations de l'Etat lui-même vont s'accélérer avec la décentralisation, qui se présente comme un concept important des théories économiques libérales. La loi du 28 mars 1982 et celles de l'année suivante ont créé les conditions institutionnelles de l'organisation décentralisée de la République. Le champ des compétences des collectivités locales s'est étendu et leur autonomie financière a été élargie et érigée en principe à l'article 72-2 de la Constitution. La décentralisation a connu une seconde phase en 2003 avec de nouveaux transferts de compétences et l'inscription dans la constitution du caractère décentralisé de l'Etat, en dépit des craintes suscitées d'ordre politique et économique, devant les risques de démembrement de l'Etat et d'explosion des impôts locaux liée à l'extension des compétences des collectivités locales et territoriales. Néanmoins, le mouvement de décentralisation a franchi de nouveaux pas en 2009, en 2010, puis en 20142015. De plus en plus les finances locales sont devenues un révélateur des sociétés modernes et un élément essentiel pour juger le courant de décentralisation à ses effets. Un saut qualitatif a été franchi, qui a conduit à une organisation locale qui s'est de plus en plus compliquée avec l'apparition et la multiplication à partir de 1999 des établissements publics de coopération intercommunale (EPCI). C'est la nature du système local ainsi que son environnement interne et externe qui se sont modifiés substantiellement

Parallèlement, les règles générales de la gestion publique ont évolué vers davantage de pouvoirs accordés aux gestionnaires publics locaux, qui en contrepartie deviennent responsables des résultats de leur gestion, dont ils doivent rendre compte. Dans ces conditions, le principe de responsabilité lié à l'exigence démocratique de protection accrue des fonds publics menace d'être vidé de son contenu en l'absence d'un système de contrôle et de sanction adéquat ${ }^{2}$.

La réorganisation du système local posait donc de nouvelles questions sur le terrain financier et de la gestion, dans la mesure où le choix des objectifs relève du seul pouvoir local au nom du principe de libre administration locale. C'est pourquoi il est devenu nécessaire de contrôler plus efficacement la gestion locale, dans un contexte devenu complexe et exposé aux risques les plus divers. La qualité de ce contrôle conditionne toute nouvelle extension de la décentralisation et l'accroissement du pouvoir financier local, qu'implique le principe de subsidiarité qui s'affirme de plus en plus. La responsabilisation corrélative des gestionnaires locaux ${ }^{3}$, qu'il est important de ne pas laisser seuls face aux difficultés de leurs tâches et aux contraintes financières nouvelles, exige de leur venir en aide en instaurant un contrôle fondé à la fois sur la transparence, l'évaluation et le conseil.

2 A. FROMENT-MEURICE et N. GROPER « La responsabilité des acteurs de la gestion publique en matière budgétaire, financière et comptable : l'heure du bilan ", in Actualité Juridique de Droit Administratif (AJDA) $\mathrm{n}^{\circ} 13 / 2005$, p. 714.

3 Qui s'est amorcée avec l'octroi de prêts et de subventions globalisés et un début de réforme de la fiscalité locale. Sur ce point, M. BOUVIER, Les finances locales, L.G.D.J., 2015, p. 233. 
L'impératif d'efficacité et l'autonomie locale croissante justifient et imposent même un contrôle approprié.

C'est ainsi que d'emblée la loi du 2 mars 1982, d'une part a substitué au contrôle de tutelle de l'Etat exercé a priori sous la forme de l'autorisation préalable du préfet en matière financière un contrôle a posteriori sur les actes des collectivités locales, et d'autre part a institué des Chambres régionales des comptes pour chaque région ou ensemble de régions. Ce mode de contrôle auquel sont d'ailleurs associés les tribunaux administratifs est conforme à la logique de la décentralisation et correspond aux exigences de l'Etat de droit ${ }^{4}$. Ce contrôle externe exercé par des organismes indépendants, susceptible d'entraîner l'annulation de certains actes ou leur mise en conformité, répond aux impératifs d'une société démocratique et à la complexification des nouveaux modes de gestion.

\section{I - LA NÉCESSITÉ CROISSANTE DU CONTRÔLE DE LA GESTION LOCALE}

Le système français du contrôle des finances publiques et de la gestion locale est en réalité éclaté entre plusieurs institutions. Il s'agit d'une part d'un contrôle administratif exercé par le ministre des finances sur le comportement des comptables publics, et d'autre part du contrôle des juridictions financières (Chambres régionales et territoriales des comptes et en appel la Cour des comptes) qui relèvent du Conseil d'Etat en tant que juge de cassation.

Les collectivités locales et territoriales sont des acteurs essentiels de la vie démocratique et économique, en matière financière en général et sur plan local en particulier. Il faut donc satisfaire à la fois l'exigence d'information correcte des citoyens et l'impératif de saine gestion des fonds publics.

\section{$\S 1$. Les fondements politiques et juridiques du contrôle}

Il ne serait pas concevable de relâcher à la fois le contrôle a priori et le contrôle a posteriori, sans remettre en cause l'exigence démocratique de vérification de l'utilisation de l'argent public. Celle-ci doit être encadrée par des règles démocratiquement établies. Il y va de la nature même du régime politique, de placer sous contrôle les décisions concernant les finances publiques. On a pu dire que «L'éclat de la démocratie est intimement lié à (la) transparence financière de ses institutions $»^{5}$.

La spécificité des finances publiques implique un régime de protection approprié. Comme il s'agit essentiellement de ressources provenant de

4 Si l'efficacité du droit réside plus dans la prévention, le système doit être compatible avec les principes de la décentralisation. Voir, J. HOUËL, « Le contrôle financier des Chambres Régionales des Comptes ", in POUVOIRS, $n^{\circ}$ 60/1992, p.143, et B. LEVALLOIS, " Quel contrôle externe pour les collectivités locales », in Revue Française de Finances Publiques (RFFP), N 85/2004, p. 11 et s.

${ }^{5}$ M. BOUVIER, op. cit., p. 7. 
prélèvements obligatoires démocratiquement autorisés, leur utilisation doit être démocratiquement fondée et justifiée aux yeux des citoyens. Cela légitime non seulement l'existence d'un droit particulier, mais aussi une protection efficace exercée notamment par un juge spécialisé disposant de compétences techniques particulières. Pour D. MIGAUD, Premier président de la Cour des comptes, « Le consentement à l'impôt étant au fondement de la démocratie, les citoyens doivent donc être convaincus de la nécessité d'y consentir, et être en mesure d'évaluer les résultats effectifs des décisions prises, mais trop souvent les résultats obtenus ne sont pas conformes aux objectifs annoncés et ne sont pas à la hauteur des moyens publics qui leur sont consacrés ${ }^{6}$. La mission des juridictions administratives en général et financières en particulier se fonde sur les articles 14 et 15 de la Déclaration des droits de l'Homme de 1789 intégrée au Préambule de la constitution ${ }^{7}$, qui valorisent aussi l'exigence d'information. Outre le contrôle externe exercé par l'Etat, les Chambres régionales et territoriales des comptes (CRTC) sont érigées par la loi en contrôleurs naturels des comptes publics locaux. Elles sont ainsi placées au centre d'enjeux majeurs qui touchent à l'autonomie locale, et doivent concilier deux logiques différentes ; l'une confiant à des organismes publics d'Etat le contrôle des finances locales, et l'autre soucieuse d'une certaine libéralisation de la gestion locale axée sur la reconnaissance de droits et de garanties véritables.

$\mathrm{Si}$ au départ les Chambres régionales comptes ont été perçues par les élus locaux comme des obstacles au fonctionnement des collectivités locales ${ }^{8}$, pour les citoyens l'important est de savoir si elles sont en mesure de vérifier que les finances locales sont correctement utilisées, et de déceler voire même d'empêcher des situations dangereuses ou malsaines pour la collectivité. Pour cela, il faut que la transparence, le contrôle et l'évaluation soient rendus possibles. Cela est devenu d'autant plus indispensable que les modes de gestion au plan local se sont modernisés, faisant appel aux partenariats et à la contractualisation. En effet, depuis la décentralisation la gestion des collectivités locales se présente comme un nouveau mode d'action publique. Elle s'est professionnalisée, «tant dans son fonctionnement stratégique de planification en matière d'investissement notamment, que dans le domaine du management de la gestion»?.

La loi organique du 21 décembre 2001 (relative aux lois de finances) qui tendait à réaliser une réforme de l'Etat, a introduit de nouvelles logiques de gestion et de contrôle. Elle a préconisé une gestion performante des finances publiques et promu une culture de l'évaluation des politiques, en transposant ces nouvelles exigences

6 Propos tenus à l'occasion de la publication du Rapport public annuel 2015 de la Cour des comptes, in Les Annonces de la Seine, 23 février 2015, p. 6.

Article 14 : « Tous les citoyens ont le droit de constater par eux-mêmes ou par leurs représentants la nécessité de la contribution publique, de la consentir librement, d'en suivre l'emploi ».

Article 15 : «La société a le droit de demander compte à tout agent public de son administration ».

8 M. MERCIER, « Du contrôle au conseil », in R.F.F.P., n 85/2004, p. 74.

9 D. LAMARQUE, «L'évolution des politiques publiques locales par les Chambres régionales des comptes : jusqu'où ?» in RFFP, n 85/2004, p. 59. Egalement, E. PORTAL, « La planification stratégique locale: pratiques et enjeux », in RFFP, $n^{\circ} 85 / 2004$, p. 107 et $\mathrm{s}$. 
au plan local. Les acteurs locaux ont donc eu recours à certaines techniques du management public, utilisant des outils destinés à définir les politiques publiques concourant au développement local. Cela a conduit les Chambres régionales des comptes à procéder à l'évaluation des politiques publiques qui ont toutes un impact local et décentralisé. Leur contrôle ne se fait plus comme à l'origine, et il a désormais intégré une dimension d'évaluation. Il s'agit donc pour elles d'assurer une information générale, complète et compréhensible des actions et des décisions engageant les finances locales. Au-delà de la seule transparence des comptes, l'information financière doit éclairer les différents choix de gestion et assurer la bonne utilisation des ressources.

Relayant la loi organique sur les lois des finances de 2001, la révision constitutionnelle de juillet 2008 a donné valeur constitutionnelle au principe selon lequel « les comptes des administrations publiques sont réguliers et sincères (et) donnent une image fidèle du résultat de leur gestion, de leur patrimoine et de leur situation financière $»^{10}$. Cette qualité de l'information est indispensable à l'exigence d'une authentique démocratie locale, permettant aux citoyens d'être informés des affaires locales et d'apprécier les décisions qui les concernent. $\mathrm{Ce}$ droit qui est le leur ${ }^{11}$, qui fait aussi partie de la libre administration des collectivités territoriales, est un élément essentiel de la démocratie locale.

L'évolution de la décentralisation et les transferts successifs de compétences posent avec acuité le problème de l'application au secteur public local des règles nationales du droit public financier édictées dans l'intérêt général des finances publiques. L'exigence de régularité et de sincérité des comptes locaux a aussi un fondement juridique communautaire, notamment dans la directive de l'Union européenne du 8 novembre 2011, qui concerne le respect par les Etats membres de leurs obligations d'éviter les déficits. Ceci conduit à évoquer les aspects proprement économiques du contrôle de la gestion locale.

\section{§ 2. Les justifications d'ordre économique du contrôle}

$\mathrm{Si}$ les finances locales ont pu être considérées comme une composante secondaire des finances publiques, leur place actuelle dans l'économie et leur influence sur l'état des finances publiques sont considérables. Par ailleurs, elles évoluent dans un contexte général en mutation permanente. Au niveau local, une multitudes d'acteurs agissent : les régions, les départements, les communes, leurs établissements publics, les sociétés d'économie mixte, les associations, les groupements d'intérêts publics, les entreprises, les banques. À cela il faut ajouter les services déconcentrés de l'Etat. Les opérateurs locaux sont sans cesse plus nombreux et plus divers, les circuits de financement se sont complexifiés, sans

10 Art. 47-2 de la constitution, introduit par la loi constitutionnelle du 28 juillet 2008, de modernisation des institutions de la cinquième République. Voir D. ADVIELLE et P. VAN HERZELE, "Vers une assurance renforcée sur la régularité et la sincérité des comptes des collectivités locales », in A.J.D.A., $n^{\circ}$ 10/2014, p.559.

11 Défini à l'article L 2141- 1 du Code général des collectivités locales (CGCT). 
oublier les liens de plus en plus étroits avec les institutions européennes. Tout cela fait que chaque collectivité locale est connectée à un ensemble structuré de réseaux, au point qu'il convient maintenant de situer les finances locales dans une perspective européenne voire mondiale ${ }^{12}$.

Dans un premier temps, l'extension des compétences locales a connu une période d'opulence, à laquelle a succédé une crise financière accompagnée par une évolution des mentalités privilégiant l'individualisme, alors que l'Etat centralisé était affaibli, notamment sous l'effet des conceptions économiques néolibérales. Après l'affirmation d'une certaine liberté de gestion des collectivités locales, des contraintes nouvelles découlant de l'internationalisation des échanges ont imposé aux Etats des politiques financières plus restrictives. L'intégration européenne s'est aussi répercutée sur les collectivités locales, confrontées à une compétition nationale et externe de plus en plus forte. L'Union européenne, après avoir instauré la monnaie unique, a obligé l'ensemble du secteur public à participer aux objectifs financiers nouveaux (respect des critères de convergence, limitation des déficits et de l'endettement publics, etc...).

Les collectivités locales participent de manière importante au développement économique, et les gestionnaires locaux sont devenus des acteurs dont les décisions cumulées ont un impact non négligeable sur les finances publiques dans leur ensemble et sur leur équilibre global. Les finances locales représentent un enjeu majeur, compte tenu de leur part dans la dépense publique totale, et de leur rôle en matière d'investissement et notamment dans le domaine social. Les médias ont souvent évoqué la situation financière parfois calamiteuse de certaines collectivités, et l'opinion publique " s'étonne que de telles situations n'ont pas pu être décelées à temps et empêchées $\rangle^{13}$. Ces derniers temps, la crise financière locale s'est aggravée, dans un contexte national et européen évolutif, fragilisé et fortement dégradé.

La montée des dépenses locales a été d'autant plus inexorable, que l'Etat n'a cessé de déléguer un nombre croissant de tâches. Il a été constaté qu'en 2009 ces dépenses représentaient $20 \%$ de l'ensemble de la dépense publique, qu'elles ont augmenté plus vite que la richesse nationale, alors que les ressources propres ne permettent de les financer que très partiellement, en dépit d'une autonomie financière des collectivités locales érigée en principe par la révision constitutionnelle intervenue en $2003^{14}$. Pour sa part, la Cour des comptes dans son

12 Voir J.M. BOLLÉ et E. NOUVEL, «Les collectivités locales et le passage à l'euro », in RFFP, $\mathrm{n}^{\circ}$ 62/1998, p. 79 et s. ; P. LAURENT, « Les villes et l'euro », eod. loc., p. 97 et s. ; J. BOUINOT, « L'euro et les disparités Interrégionales des richesses », eod. loc., p. 107 ; B. CHEVAUCHEZ, « Les effets de la construction européenne sur notre système de finances publiques ", in R.F.F.P., n 68/1999, p. 40 et s.

13 J. HOUËL, op. cit., p. 142.

14 Ce sont les constats du Comité pour la réforme des collectivités locales, dans son rapport de mars 2009 qui a noté que la question des finances locales est " au cœur de toutes les réformes » : cité par M. BOUVIER, Les finances locales (précité), p. 40. La loi organique du 29 juillet 2004, relative à l'autonomie financière locale et prise en application de l'article 72-2 de la constitution, a défini les éléments de l'autonomie financière permettant de déterminer la notion de « ressources propres ». Cellesci ont subi l'impact négatif des allégements fiscaux que l'Etat a dû consentir récemment pour soulager la «pression fiscale » pesant sur les citoyens et les entreprises. 
Rapport publié le 14 octobre 2014 et consacré aux finances locales, a souligné leur inquiétante dégradation ${ }^{15}$. La dette des administrations locales a atteint 182 milliards d'euros, soit $10 \%$ du montant total de la dette publique de l'époque (qui dépassera les 2.200 milliards d'euros avant la fin de 2015). Les dépenses des collectivités locales et de leurs services annexes représentent $60 \%$ des dépenses de l'Etat et près des $3 / 4$ de la formation brute du capital fixe des administrations publiques ${ }^{16}$. Les budgets locaux pèsent de plus en plus lourd dans les dépenses (250 milliards d'euros), dans les recettes et la fiscalité (100 milliards d'euros représentant $50 \%$ des ressources locales).

Cette évolution explique que l'on aille aujourd'hui vers un renforcement du pilotage par l'Etat du système financier public, pour éviter son éclatement. C'est la crise économique et financière qui dure toujours en 2015, qui a replacé l'Etat au premier plan, ce qui contribue à transformer les rapports avec les collectivités locales et à redéfinir leur pouvoir financier. Cela est conforme au rôle de l'Etat, qui est chargé de prélever les impôts et de veiller à la bonne utilisation de la part des recettes fiscales attribuée aux collectivités locales. Tout en respectant la libre administration locale, ce contrôle légitime doit être simple, efficace et utile.

\section{II - LES MODALITÉS DU CONTRÔLE DE LA GESTION LOCALE PAR LES CHAMBRES RÉGIONALES ET TERRITORIALES DES COMPTES}

Les finances publiques n'étant pas seulement de l'économie ou de la gestion, mais n'étant pas non plus uniquement du droit, cela « justifie la spécificité des Chambres régionales des comptes, la diversité de leurs missions et la variété des contrôles exercés $\rangle^{17}$. Au sein du système dynamique et évolutif que sont les finances locales, le contrôle doit donc combiner normativisme et gestion, ce qui suppose une certaine attitude intellectuelle et une formation à la complexité qui caractérise les sociétés modernes. Le seul examen de la légalité a posteriori n'étant pas suffisant et le contrôle financier impliquant d'autres approches, ce sont donc des missions différentes qui sont confiées aux Chambres régionale des comptes, ce qui en fait une juridiction particulière. Celle-ci ne doit pas être perçue comme une institution répressive, mais plutôt comme un élément essentiel d'une bonne gestion publique, assumant un contrôle exercé principalement a posteriori, mais impliquant «d'autres approches, préventives, d'analyses et de propositions $»^{18}$.

15 J.M. PASTOR, « Les pistes de la Cour des comptes pour maîtriser la dépense locale », in AJDA, $\mathrm{n}^{\circ} 35 / 2014$, p. 1973.

16 Sur ces divers points, M. BOUVIER, Les finances locales (précité), p. 12.

17 M. BOUVIER, « Repenser et reconstruire les finances publiques de demain », in RFFP, $n^{\circ}$ 85/2004, p. 4.

${ }^{18}$ M. BOUVIER, Les finances locales (précité), p. 137. 


\section{§ 1. La nature spécifique de la juridiction financière}

La création de 22 Chambres régionales des comptes en 1982, visait d'une part à réduire les pouvoirs du préfet dans l'appréciation des comptes des collectivités locales, et d'autre part à soulager une Cour des comptes bien encombrée ${ }^{19}$. Mais surtout il fallait, conformément à l'esprit de la décentralisation, confier le contrôle des actes financiers des collectivités locales à une autorité autonome du pouvoir politique (central ou local), et offrant des garanties suffisantes.

\section{A/ LA CHAMBRE RÉGIONALE ET TERRITORIALE DES COMPTES : ORGANISATION ET STATUT DES MEMBRES}

En principe recrutés par la voie de l'Ecole Nationale d'Administration (comme les magistrats administratifs), les magistrats financiers sont aussi recrutés par concours de recrutement direct ${ }^{20}$. Les Chambres régionales des comptes (ainsi que les Chambres territoriales des comptes pour l'Outre-Mer) sont présidées par un membre de la Cour des comptes (conseiller maître ou conseiller référendaire) et composées de magistrats inamovibles. Les plus importantes sont divisées en sections.

L'organigramme type d'une chambre régionale comprend, outre le président et un secrétaire général, un ministère public composé d'un Procureur et d'un assistant, un secrétariat général, un service du greffe et des archives, un service de documentation, chaque section comprenant un président, des membres et des assistants de vérification ${ }^{21}$.

Les membres des CRTC sont soumis aux obligations des fonctionnaires, auxquelles s'ajoutent celles qui résultent de leurs propres statuts ${ }^{22}$. Les principales règles à respecter sont l'indépendance, l'impartialité, la neutralité, l'intégrité, la discrétion, le secret, la prévention des conflits d'intérêts afin de séparer nettement les travaux de vérification des activités personnelles ou antérieures.

Toutes ces règles sont reprises dans une Charte de déontologie entrée en vigueur en 2006. Il y est prévu que dans le cadre de leurs activités privées (d'ordre politique, philosophique ou confessionnel) les magistrats financiers ne doivent pas mettre en avant leur appartenance à l'institution ou à ses travaux. Il y est également mentionné, que la nature des activités extérieures ne doit pas nuire à la réputation de l'institution, et que leur exercice est soumis à l'avis préalable et éventuellement à l'autorisation du collège de déontologie. De même, un délai de 3 ans doit s'écouler avant de pouvoir participer au contrôle d'un organisme dans

19 C. DEBBASCH, Droit administratif, Economica, $6^{\text {ème }}$ éd, p. 338.

${ }^{20}$ Ce système mis en place par la loi Sauvadet a été pérennisé à la demande du premier président de la Cour des comptes : cf. D. MIGAUD, «Il n'y a pas d'affaiblissement du contrôle des Chambres régionales des comptes », in AJDA, n 9/2012, p. 461.

${ }^{21}$ D'après le schéma proposé par M. BOUVIER, Les finances locales (précité), p. 255.

22 Outre certaines incapacités et incompatibilités, cela concerne le « serment professionnel ... de bien et fidèlement remplir ses fonctions, de garder le secret des délibérations et de se comporter en tout comme un bon et loyal magistrat ». 
lequel des responsabilités ont été antérieurement exercées. La règle du « déport » est imposée, lorsque « des éléments personnels sont susceptibles de porter atteinte à leur impartialité $»^{23}$. La loi du 12 mars 2012 interdit aussi aux magistrats tout détachement ou mise à disposition dans une collectivité ou un organisme situés dans le ressort de la Chambre régionale des comptes à laquelle ils ont appartenu.

\section{B/ LES ÉVOLUTIONS RÉCENTES.}

Une réflexion sur la réforme des chambres régionales des comptes a débuté en $2005^{24}$, axée sur la responsabilité des gestionnaires publics en général. Dans ses différentes phases successives elle a suscité les oppositions de certains parlementaires (eux-mêmes parfois gestionnaires locaux) mais aussi de nombreux magistrats financiers. En effet, un projet de loi du 28 octobre 2009 prévoyait la disparition des Chambres régionales des comptes et leur absorption par la Cour des comptes, pour en faire de simples chambres en son sein. C'est pourquoi la réforme n'a pas pu être votée d'un seul bloc et a été tronçonnée en trois lois successives : celles du 29 juillet, du 13 et du 28 décembre 2011. Finalement, il a été décidé de réduire de 22 à 15 le nombre des Chambres régionales et territoriales des comptes, pourtant conçues initialement comme juridictions de proximité, et de redéfinir leur ressort territorial devenu interrégional pour certaines. Le but a aussi été de mieux répartir la charge de travail collective, de rendre plus présentes et plus efficaces certaines petites structures, de favoriser le travail en équipe, de faire vivre plus encore la collégialité, et de permettre la spécialisation au sein des juridictions.

La loi du 13 décembre 2011 a dû être validée par le Conseil constitutionnel, et la réorganisation envisagée (décret du 23 février 2012) a été confirmée par le Conseil d'Etat (C.E. 20 mars 2013, Association des magistrats des Chambres régionales et territoriales des comptes), qui a rappelé que la détermination du nombre, du siège et du ressort de ces juridictions était bien de la compétence du gouvernement, qu'il n'y a pas de droit acquis au maintien d'une réglementation, et que la sécurité juridique n'était en rien entachée en l'absence d'effet rétroactif de cette réorganisation ${ }^{25}$.

\section{C/ L'ORIGINALITÉ MAINTENUE DU JUGE FINANCIER LOCAL}

Le contrôle de régularité des actes et opérations de nature financière, est une composante du contrôle de légalité exercé sur l'action administrative des collectivités locales et de leurs établissements publics. Cela pose le problème de

${ }^{23}$ Sur tous ces points, M.T. CORNETTE, « La Charte de déontologie des juridictions financières », in AJDA, n¹6/2012, p. 880 et $\mathrm{s}$.

24 Elle portait au début sur la responsabilité des gestionnaires publics : se reporter à la Tribune de $\mathrm{P}$. SEGUIN et H. GISSEROT, in AJDA, n¹3/2005, p. 689 et à M.C. de MONTECLERC, « La réforme des juridictions financières est lancée », in AJDA, n³6/2009, p. 1978.

25 Sur cette réforme, S. DAMAREY, «L'acte (II) manqué de la réforme des juridictions financières », in AJDA, n 6/2012, p. 318 et s. ; L. FERMAUD, « Le point final apporté à la réforme des juridictions financières "), in AJDA, $n^{\circ}$ 28/2013, p. 1974 et $\mathrm{s}$. 
l'articulation du rôle des Chambres régionales des comptes avec la fonction de juge de droit commun attribuée aux tribunaux administratifs, érigés en contrôleurs naturels des actes locaux par les lois de décentralisation. C'est aussi la nature de l'institution qui est en cause. Car dans l'accomplissement du contrôle externe conféré à la Chambre régionale des comptes, la fonction de juge coexiste avec celle de conseiller, étant entendu que la nature de juridiction qui lui est reconnue constitue une garantie d'indépendance sans en faire un organisme chargé de sanctionner les élus locaux. Pour Daniel CHABANOL, un tel juge « ne peut pas se contenter d'exercer un contrôle formel, car il ne suffit pas qu'une dépense soit formellement régulière pour qu'elle soit forcément légale $\gg^{26}$. En effet, en intervenant sur les comptes des comptables publics locaux, le juge financier se comporte en contrôleur de la légalité intrinsèque des opérations et actes examinés. Or, les comptables publics locaux ne font qu'exécuter les ordres de dépense ou de recette après avoir vérifié l'exactitude des calculs et les justificatifs qui leur sont présentés. Mais comme ils engagent leur responsabilité personnelle devant le juge des comptes, ils ont tendance à vérifier la légalité au fond des décisions de ceux qui ont ordonné lesdites opérations. C'est là une dérive qui, d'une part conduit le juge administratif à rappeler aux comptables publics locaux les limites de leur contrôle ${ }^{27}$, et qu'il ne peut y avoir concurrence ou double emploi entre les missions du juge administratif et celles du juge financier. D'autre part et s'agissant des Chambres régionales des comptes, le Conseil d'Etat en tant que régulateur suprême du contrôle de légalité veille à faire respecter la séparation entre leur fonction juridictionnelle et leur activité de conseil, dans l'exercice du contrôle externe qui leur est dévolu ${ }^{28}$.

Il faut donc en déduire avec D. CHABANOL, que les Chambres régionales des comptes, comme la Cour des comptes, sont « les juges d'une légalité spécifique » ${ }^{29}$, que la mission de contrôle juridictionnel des comptes et celle de conseil sont et doivent rester distinctes ${ }^{30}$, mais que le contrôle pour être efficace comporte une dimension de conseil. Cela confirme qu'en matière de finances publiques deux logiques sont à l'œuvre : d'un côté la logique politique et juridique classique procédant d'une tradition qui privilégie la capacité décisionnelle des gestionnaires locaux en matière financière, et d'un autre côté la logique de gestion répondant à des impératifs économique et qui valorise l'expertise technique ${ }^{31}$. Les Chambres régionales des comptes, par leur nature et par les modalités de leur contrôle,

${ }^{26}$ D. CHABANOL, «Les Chambres régionales des comptes, un autre juge de la légalité ? », in RFFP, $\mathrm{n}^{\circ} 85 / 2004$, p. 52.

27 C.E., 10 février 1997, Ibo ; C.E., 8 décembre2000, Kammerer.

28 C.E., Ass., 23 février 2000, Société Labor Métal.

${ }^{29}$ D. CHABANOL, op. cit., p. 52.

${ }^{30}$ A. PICHON, « Le contrôle et le conseil : une cohabitation problématique », in RFFP, n 85/2004, p. 87.

${ }^{31}$ Sur ce point, M. BOUVIER «Repenser et reconstruire les finances publiques de demain » (précité), p. 7. 
réalisent cette fusion des impératifs juridiques et des considérations économiques et de gestion.

\section{§ 2. La diversité des missions dévolues aux juridictions financières.}

Ce qui vient d'emblée à l'esprit à propos de l'action locale, c'est la notion de contrôle de régularité exercé par les instances administratives et juridictionnelles. S'agissant des finances locales, le contrôle fait intervenir à la fois les représentants de l'Etat (les préfets) et les Chambres régionales des comptes ${ }^{32}$. Cela correspond à la nécessité de veiller à l'application correcte des normes conformément à l'Etat de droit, avec le souci de la transparence du fonctionnement institutionnel propre à la démocratie. La préoccupation de mieux gérer a aussi progressivement conduit à des pratiques managériales empruntées aux entreprises, et cette évolution a débuté pour l'Etat lui-même avec le processus de réforme engagé en 2001 avec la loi organique relative aux lois en finances (LOLF). La recherche de la qualité et de la performance de la gestion publique, s'inscrit donc dans un corps de règles générales et de dispositions propres aux finances publiques. Cette nouvelle logique introduite dans la gouvernance financière publique moderne, s'applique tout autant à l'Etat qu'aux collectivités locales et territoriales. C'est pourquoi, à côté du contrôle juridictionnel qui leur a été initialement attribué à titre principal, les juges financiers se sont vu confier des missions essentiellement administratives et à caractère non juridictionnel portant à la fois « sur la régularité des opérations et sur la bonne gestion financière, ce qui correspond à un contrôle de gestion $»^{33}$. Pour concilier les exigences démocratiques et juridiques construites dans un autre contexte et les impératifs de la gestion moderne des finances publiques locales, les Chambres régionales des comptes exercent un triple contrôle : un contrôle proprement juridictionnel à travers le jugement des comptes, ainsi qu'un contrôle budgétaire et un contrôle de gestion.

\section{A/ LE CONTRÔLE JURIDICTIONNEL SUR LES COMPTES DES COLLECTIVITÉS LOCALES.}

Il a été rappelé que le préfet est responsable en premier lieu du contrôle de légalité des actes des autorités locales ${ }^{34}$. Dans le domaine financier, c'est le comptable public local qui représente un premier rempart contre les irrégularités.

\section{$1 \%$ Le champ de compétence des Chambres régionales des comptes}

Si le préfet est en droit de s'assurer de la sincérité des inscriptions au budget local des recettes et des dépenses, c'est le comptable public local (avec sa

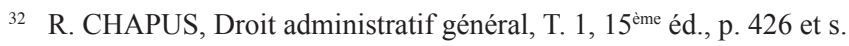

33 L. SAÏDJ, «La loi organique du 19 août 2001 sur les lois de finances : quels enseignements pour les collectivités locales? », in RFFP, n 85/2004, p. 46.

34 Le juge administratif contrôle le respect par le préfet de l'obligation de motiver ses décisions : C.E., 10 octobre 1990, Préfet du Val d'Oise. 
hiérarchie) qui constitue un premier échelon du contrôle externe de la régularité des comptes.

\section{a) Le principe de la séparation des ordonnateurs et des comptables.}

Si dans le secteur privé et au sein de l'entreprise le comptable est interne, alors que la certification par les commissaires aux comptes est externe et porte sur un compte unique présenté par les dirigeants de l'entreprise, il en va différemment dans le secteur public. L'exécution des opérations financières des collectivités publique est dominée dans le système français par un principe : celui de la séparation des ordonnateurs et des comptables, qui distingue clairement les responsabilités à chaque stade de la dépense publique ${ }^{35}$. Les ordonnateurs sont ceux qui donnent « l'ordre » d'encaisser une recette ou de décaisser une dépense : ce sont les divers dirigeants, maires, ou présidents des assemblées départementales ou régionales. Les comptables sont des agents de la Direction Générale des finances publiques (au sein du ministère des finances), qui procèdent au paiement des dépenses et au recouvrement des recettes. Ils établissent une comptabilité complète des opérations de la collectivité, qui en fin d'exercice donne lieu à la production du compte de gestion, qui est transmis à la collectivité publique concernée. Son assemblée ou organe délibérant se prononce ensuite sur le compte administratif qui lui est présenté par l'ordonnateur pour approbation. Cette double comptabilité permet aux élus locaux de vérifier la conformité des opérations effectuées par l'ordonnateur ${ }^{36}$

Le comptable public qui exerce déjà un contrôle de légalité sur les ordres de paiement peut en suspendre l'exécution par une décision motivée notifiée à l'ordonnateur, qui peut cependant lui adresser un ordre de réquisition qui a pour effet de transférer sur l'ordonnateur la responsabilité du paiement ${ }^{37}$. Ce faisant, le comptable joue un rôle d'alerte et apporte aux gestionnaires locaux la sécurité dont ils ont besoin. À la fois extérieur et proche, il a une connaissance de la collectivité et de son environnement, qui lui permet d'exercer un contrôle proportionné à l'appréciation des enjeux et des risques. D'autant que la loi de 2001 (LOLF) a innové, puisque tout en rappelant son rôle de caissier et de teneur de comptes, elle a fait du comptable public un garant de leur sincérité et du respect des procédures.

35 Cette division entre ces deux fonctions a été codifiée par l'ordonnance du 14 septembre 1822 et reprise dans le décret du 7 novembre 2012 (art. 8). Ce texte rappelle (art. 9) que « les fonctions d'ordonnateur et de comptable sont incompatibles ».

36 F. LOGEROT, « Le contrôle externe des collectivités locales : actualité et perspectives », in RFFP, $n^{\circ} 85 / 2004$, p. 17 ; P.H. WATINE, « Le système français de contrôle des comptes des collectivités locales : quelles évolutions ? », in RFFP, $n^{\circ}$ 85/2004, p. 19 ; A. LAMBERT, « Les Chambres régionales des comptes doivent aussi devenir des acteurs de la modernisation », in RFFP, ${ }^{\circ}$ 85/2004, p. 92 ; F. ADVIELLE et P. VAN HERZELE, «Vers une assurance renforcée sur la régularité et la sincérité des comptes des collectivités territoriales », in AJDA, $n^{\circ}$ 10/2014, p. 558 ; M. BOUVIER, Les finances locales (précité), p. 240 et $\mathrm{s}$.

37 C. DEBBASCH, op. cit., p. 338 et G. PEISER, Droit administratif général, Dalloz, 25éd, p. 128. Egalement, P. JAMET, « De la proximité du comptable public », in RFFP, n 85/2004, p. 27. 
Le principe de séparation évoqué précédemment s'en trouve donc assoupli « pour laisser place à une collaboration $»^{38}$.

\section{b) Le périmètre du contrôle du juge financier.}

Le comptable, premier contrôleur externe, est lui-même contrôlé par la Chambre régionale des comptes qui est donc " un contrôleur du contrôleur externe $»^{39}$. Elle statue sur les comptes des collectivités locales et territoriales, de leurs établissements publics, des services annexes, y compris parfois sur les opérations réalisées par des associations entretenant des liens étroits avec certaines collectivités publiques de leur ressort ${ }^{40}$. Dans l'exercice de son contrôle juridictionnel, la Chambre régionale des comptes intervient dans les mêmes formes et avec les mêmes pouvoirs que la Cour des comptes. Cependant, la loi du 15 décembre 2011 modifiant celle du 21 décembre 2001, a institué un régime spécifique pour les communes et les établissements publics de coopération intercommunale (EPCI) de moindre importance ${ }^{41}$.

Le contrôle des comptes du comptable public consiste à vérifier la correction des écritures et la bonne application des règles de la comptabilité publique, à partir des informations fournies par l'ordonnateur. En cas de non-conformité des comptes, la responsabilité personnelle du comptable public pourra être engagée. Cela incite à plus de vigilance dans la tenue des comptes et des pièces justificatives (par exemple celles afférentes au paiement d'un marché public). Quant aux citoyens, aux partenaires des collectivités locales, aux pouvoirs publics, il leur est possible d'être fixés dans des délais raisonnables sur la fiabilité des comptes.

\section{$2 \%$ Les aspects procéduraux}

\section{a) La situation ancienne.}

Selon la formule traditionnelle, « le juge des comptes juge les comptes et non les comptables ». La procédure classique était d'ordre public et caractérisée par un mécanisme d'autosaisine, elle était écrite, inquisitoire et secrète. Dans un premier arrêt, le juge faisait connaître ses reproches en enjoignant au comptable d'y répondre. C'est dans une seconde décision que le juge financier statuait définitivement en prononçant la décharge (ou quitus) ou le « débet » qui mettait en jeu la responsabilité pécuniaire personnelle du comptable. Mais en réalité, la

\footnotetext{
38 L. SAÏDJ, op. cit., p. 44.

39 Il s'agit d'un contrôle externe de deuxième degré. J. BELLE, « L'Oxymore », in RFFP, n 85/2004, p. 25 .

40 C'est la question des « comptables de fait», qui ne sera pas évoquée dans le cadre de cette étude, mais qui constitue un aspect important du contrôle de la gestion locale.

41 Ici, le champ des compétences directes des Chambres régionales des comptes est plus restreint. Il est procédé à un apurement administratif. Cela concerne les communes dont la population n'excède pas 5.000 habitants et dont les recettes de fonctionnement sont inférieures à 3.000.000 d'euros. Ces limites sont portées à dix mille habitants et 5 millions d'euros pour les EPCI. Ces collectivités sont contrôlées par l'administrateur général des finances publiques, sauf si la responsabilité du comptable public est engagée et sur laquelle seule la Chambre régionale des comptes est alors habilitée à statuer. Voir M. BOUVIER, Les finances locales (précité), p. 258.
} 
décision provisoire est apparue comme « préjugeant » l'issue de l'affaire, sans compter que l'instruction se déroulait hors la présence du comptable et que les audiences n'étaient pas publiques ${ }^{42}$. C'est pourquoi des premiers aménagements procéduraux ont été introduits avec la loi du 29 janvier 1993 prévoyant l'audition, suivie du décret du 23 août 1995 instituant l'audience publique en matière d'amende. Puis la loi du 21 décembre 2001 sur la Cour et les Chambres régionales des comptes a mis en place des procédures homogènes tout en renforçant leur caractère contradictoire ${ }^{43}$

Le Conseil d'Etat, à qui il revenait de tracer les limites de l'applicabilité de l'article 6-1 de la Convention européenne des droits de l'Homme aux juridictions administratives, y compris celles spécialisées en matière financière, distinguait à propos de ces dernières, leurs activités purement juridictionnelles et celles qui n'avaient pas ce caractère. Ainsi, pour les activités juridictionnelles, il a considéré que les stipulations de l'article 6-1 s'appliquaient à propos des amendes, qui sont considérées comme relevant de la notion d' "accusations en matière pénale » au sens de la Convention. Dans ce cas, les exigences du procès équitable devaient être respectées, notamment celles relatives à l'audience publique $\gg^{44}$. Mais les juges du Palais Royal rappelaient aussi l'existence des règles internes s'imposant aux juges financiers ${ }^{45}$. Celles-ci correspondaient en fait aux exigences de l'article 6-1, sans référence formelle à ses dispositions, et découlant plutôt des règles générales de procédure ${ }^{46}$. En conséquence et d'un point de vue formel, le Conseil d'Etat considérait que l'article 6-1 ne s'appliquait pas aux activités de nature non juridictionnelle ${ }^{47}$.

\section{b) Les réformes procédurales récentes}

C'est très progressivement que le système de contrôle juridictionnel s'est mis en conformité avec les exigences de la Convention européenne des droits de l'homme. Le juge de Strasbourg s'était par exemple prononcé sur l'application de l'article 6-1 dans un arrêt du 7 octobre 2003, Mme Richard Dubarry c/France, et pour la première fois à propos de la procédure de gestion de fait. Son arrêt du 13 janvier 2004, Martinie c/France a été rendu dans le domaine du jugement des

42 M. LASCOMBE et X. VANDENDRIESSCHE, « Réforme des juridictions financières : Acte I », in AJDA, n 41/2008, p. 2274 et $\mathrm{s}$.

43 Ce texte a également aménagé la procédure de la gestion de fait et défini plus précisément l'examen de la gestion, pour donner aux élus locaux des garanties supplémentaires. Sur ces points ; F. LOGEROT, op. cit., p. 16 ; A. LEYAT, « La Cour des comptes : juridiction retrouvée ? », in AJDA, n 42/2009, p. 2313.

44 C.E., 16 novembre 1998, SARL Deltana et M. Perrin.

45 Cela visait certains principes généraux du droit comme l'impartialité et le respect des droits de la défense : C.E., 23 février 2000, Société Labor Métal (précité).

46 C.E., 27 octobre 2000, Mme Michaux - Chevry et M. Madinécouty.

47 Cela concerne l'examen de la gestion et les observations formulées par les Chambres régionales des comptes, qui ne fait pas grief et ne sont pas susceptibles de recours devant le juge administratif : C.E., 8 février 1999, Commune de La Ciotat. Ne relèvent pas non plus de l'art. 6-1, les procédures de contrôle des actes budgétaires, car les avis rendus ne sont pas des actes juridictionnels, mais tout au plus parfois des actes administratifs susceptibles sous certaines conditions d'être passibles du recours pour excès de pouvoir : C.A.A., Nantes, 2 juin 2000, Caisse d'épargne et de prévoyance du Centre Val de Loire. 
comptes des comptables ${ }^{48}$. L'évolution s'est accentuée avec l'arrêt du 12 avril 2006, Martinie c/France, qui a confirmé la soumission des juridictions financières françaises aux dispositions de l'article 6-1. C'est ensuite et sous la pression de cette jurisprudence, que la loi du 28 octobre 2008 et le décret du 19 décembre 2008 ont révisé le droit processuel applicable au jugement des comptes des comptables publics, en adoptant la norme européenne du procès équitable fondée sur la théorie des apparences. Cette révolution juridique a fait basculer le juge financier dans une nouvelle philosophie du contrôle : l'audience publique est généralisée, la procédure est plus contradictoire, l'accès des parties au dossier est consacré, le magistrat instructeur ne participe plus au délibéré, le juge est ainsi mieux en mesure de décider en connaissance de cause ${ }^{49}$. Non seulement les juges financiers se sont adaptés facilement à ces " innovations ", car même sans texte certaines d'entre elles étaient déjà appliquées, mais la procédure s'est trouvée modernisée par la stricte séparation des fonctions de poursuite, d'enquête et de jugement. Ces trois moments de l'instance relèvent de trois autorités différentes au sein de la juridiction.

Depuis le $1^{\text {er }}$ janvier 2009, la première phase purement administrative assortie de garanties (information des parties et contradiction), consiste pour un rapporteur à établir en toute indépendance un rapport qui précède l'intervention de la juridiction. C'est ensuite le ministère public qui décide seul de l'orientation à donner. C'est cela qui a changé la nature du contentieux des comptes, et qui atténue le caractère d'ordre public et systématique du contrôle juridictionnel classique. C'est le "Parquet », autorité unique distincte de la juridiction, qui dispose du monopole des poursuites, en introduisant l'instance par un réquisitoire fondé sur le rapport d'un magistrat indépendant. C'est ce réquisitoire du Procureur financier et lui seul qui déclenche la phase contentieuse, en assignant au juge des comptes les limites de son intervention.

Si aucun élément ne permet de mettre en jeu la responsabilité pécuniaire personnelle du comptable, le dossier est transmis au président de la formation de jugement. Celui-ci a le choix, entre rendre immédiatement une ordonnance de décharge s'il considère qu'aucune charge ne peut être relevée, ou bien en cas de doute et en présence de lacunes dans le dossier il peut aussi demander un rapport complémentaire (avec nouvelle intervention du ministère public), qui conduira soit à une ordonnance de décharge (ou quitus), soit au déclenchement de la procédure de jugement.

Si un élément permet de mettre en jeu la responsabilité du comptable, le Procureur saisit la formation de jugement, la procédure écrite et contradictoire est

48 J.Y. BERTUCCI, « Les juridictions financières et l'article 6-1 de la Convention européenne des droits de 1'Homme », in RPPP, n 85/2004, p. 104, ; A. POTTEAU, « Le jugement des comptes confronté aux droits processuels de la Cour européenne des droits de l'homme », in Revue Française de droit administratif, 2004, p. 378.

49 G. MILLER, « Les nouvelles règles de procédures juridictionnelles devant les Chambres régionales des comptes - Premier bilan d'une pratique », in AJDA, n 31/2010,p. 1753 et s. 
alors ouverte avec débats en audience publique devant la formation collégiale de jugement.

Qu'il y ait ou non des charges, le juge intervient en toute hypothèse, ce qui justifie de dire que « le caractère d'ordre public demeure $»^{50}$.

\section{$3 \%$ Le régime de responsabilité du comptable public et ses particularités}

C'est en définitive la Chambre régionale des comptes qui décide, même si elle n'a plus l'initiative de la procédure. Les garanties de qualité du jugement données aux parties et l'obligation de procéder à l'examen rigoureux des pièces comptables, permettent de dire que le contrôle juridictionnel exercé conduit à un jugement des comptes, mais aussi du comptable, dès lors que celui-ci a toutes possibilités de présenter ses arguments ${ }^{51}$. En outre, reconnaître que le jugement des comptes relève de l'article 6-1 de la $\mathrm{CEDH}$, c'est faire automatiquement du juge financier « un juge ayant plénitude de juridiction et libéré de la seule observation des éléments matériels du compte ${ }^{52}$. Pour toutes ces raisons et eu égard aux évolutions qui se sont produites, « Il est temps de mettre fin à la fiction multiséculaire et quelque peu hypocrite du jugement des seuls comptes et non du comportement du comptable lui-même $"{ }^{53}$. On peut se demander si la relation entre l'ordonnateur et le comptable ne devrait pas elle-même évoluer, tant la séparation des deux fonctions s'atténue. Sur ce point cependant la loi n'a rien changé, et ni le comptable ni le juge des comptes ne peuvent exercer un contrôle de légalité sur les actes de l'ordonnateur ${ }^{54}$. Ce qui n'a pas changé non plus c'est le schéma contentieux, qui prévoit que les jugements rendus en première instance par les Chambres régionales des comptes relèvent toujours en appel de la Cour des comptes dont les arrêts relèvent eux-mêmes en cassation du Conseil d'Etat. Il convient toutefois de rappeler, que les décisions des juges des comptes, dont l'exécution fait aussi partie du " procès équitable » au sens de l'article 6-1 de la $\mathrm{CEDH}$ et « bien que revêtus de la formule exécutoire, ne s'exécutent que dans les limites que détermine le ministre chargé du budget, dans le cadre de la procédure de remise gracieuse $»^{55}$.

En principe le juge se borne à ordonner le rétablissement des comptes, et il peut obliger le comptable à s'acquitter d'une somme fixée en tenant compte des circonstances de l'espèce et qui ne peut dépasser un montant maximum fixé par décret. Cependant, la loi de finances rectificative du 28 décembre 2011 a fait évoluer le régime de responsabilité des comptables publics. Désormais, il faut

50 M. LASCOMBE et X. VANDENDRIESSCHE, op. cit., p. 1758.

51 Ibidem, p. 2279.

52 S. FLIZOT, « La responsabilité financière des gestionnaires publics en Europe », in AJDA, $\mathrm{n}^{\circ}$ 13/2005, p. 712.

${ }_{53}$ A. LEYAT, « La Cour des comptes : juridiction retrouvée? », in AJDA, n 42/2009, p. 2313.

54 C.E., 5 février 1971, Ministre de l'économie et des finances c/Balme ; C.E., 8 février 2012, Ministre du budget, des comptes publics et de la réforme de l'Etat.

${ }_{55}$ M. LASCOMBE et X. VANDENDRIESSCHE, op. cit., p. 2277. 
distinguer selon que le manquement n'a pas causé de préjudice financier à la personne publique concernée, ou qu'un tel préjudice peut être établi. Si tel est le cas, le comptable public doit verser la somme correspondante de son argent personnel. Le Conseil d'Etat a précisé la notion de préjudice financier et admis que la présomption qui y est attachée peut être renversée ${ }^{56}$.

Toutefois il faut ajouter, d'une part, que successivement la loi du 28 décembre 2001 a ramené à 10 ans le délai de prescription prévu par le Code Civil (30 ans) et que la loi du 28 octobre 2008 relative à la Cour des comptes et aux Chambres régionales des comptes l'a fixé à 5 ans, ce qui constitue « une modernisation bienvenue pour les comptables mais aussi pour le juge des comptes $»^{57}$. D'autre part, le ministre chargé du budget peut là encore utiliser son pouvoir de remise gracieuse en cas de préjudice financier dépassant un certain montant ${ }^{58}$

\section{B/ LE CONTRÔLE ADMINISTRATIF EN MATIÈRE BUDGÉTAIRE.}

Ce second type de contrôle est exercé a priori et en liaison étroite avec le préfet.

\section{$1 \%$ La portée et la nature du contrôle budgétaire.}

Il concerne toutes les collectivités locales situées dans le ressort territorial des Chambres régionales des comptes, ainsi que leurs groupements et les établissements publics qui leur sont rattachés (hôpitaux, collèges, lycées), mais potentiellement aussi les sociétés d'économie mixte locales, les associations subventionnées par les collectivités locales, les chambres de commerce et d'industrie ou les chambres des métiers ${ }^{59}$. Aujourd'hui, c'est le fonctionnement comptable et aussi budgétaire des collectivités locales et territoriales qui obéit à des règles proches de celles applicables aux entreprises, et le domaine budgétaire est à hauts risques non seulement pour les finances locales mais encore pour les finances publiques dans leur ensemble. C'est pourquoi la fourniture régulière de données budgétaires fiables et actualisées est indispensable à leur suivi immédiat et en temps opportun, car il faut pouvoir corriger vite une situation financière compromise.

La Chambre régionale des comptes ne rend ici aucun jugement et ne transmet que des avis au préfet. Ce partenariat entre le préfet et la Chambre régionale des comptes est encouragé, dès lors que le premier a des doutes sur la régularité ou la

56 C.E., Section, 27 juillet 2015, Ministre délégué chargé du budget c/ Parquet général près la Cour des Comptes ; s'agissant d'un cas de non recouvrement d'une créance fiscale dans le délai prescrit, étant entendu que le juge des comptes doit tenir compte de l'éventuelle insolvabilité du débiteur pour apprécier si le comptable public a causé un préjudice financier. Egalement, Cour des comptes, 11 juin 2015, Ecole Nationale de la Magistrature, statuant qu'en matière de dépenses de personnel irrégulièrement versées, le préjudice financier s'apprécie en principe au moment du paiement.

57 F. ADVIELLE et P. VAN HERZELE, « Le juge des comptes et le préjudice financier », in AJDA, n $35 / 2014$, p. 1987

58 S. DAMAREY, op. cit., p. 320, qui précise que ce montant « est fixé au double du montant de l'amende qui peut être infligée en l'absence de préjudice financier, montant fixé par décret en fonction du niveau de garantie que le comptable public est tenu de constituer lorsqu'il entre en fonction ».

59 Ce contrôle est susceptible de s'exercer sur plus de 70.000 comptabilités : M. BOUVIER, Les finances locales (précité), p. 256. 
sincérité des comptes. Le contrôle des actes budgétaires par la Chambre régionale des comptes sur saisine du préfet donne lieu à des avis et à des propositions, après instruction et délibération collégiale, ce qui leur donne une valeur d'expertise technique. Le préfet n'est certes pas tenu par ces propositions, et il peut s'en écarter en motivant sa décision qui peut toujours être contestée par la collectivité devant le tribunal administratif. En pratique, le contrôle budgétaire s'exerce sans trop de problèmes, car les préfets agissent avec un esprit de conciliation et l'intervention de la Chambre régionale des comptes est perçue comme une garantie sérieuse contre tout arbitraire étatique.

\section{$2 \%$ Les compétences des Chambres régionales des comptes}

Ce contrôle porte sur l'élaboration et l'exécution du budget. Il est limité à quatre situations, sachant que la Chambre régionale des comptes ne peut se prononcer dans les trois premiers cas que si elle est saisie par le préfet.

\section{a) Le vote tardif du budget}

Les budgets locaux doivent être votés avant le 31 mars et être transmis au préfet dans les 15 jours suivant la date limite prévue pour leur adoption, avant de devenir exécutoires de plein droit. Si les délais ne sont pas respectés, ou si des irrégularités sont constatées par le préfet, celui-ci doit saisir la Chambre régionale des comptes (ou le tribunal administratif dans certains cas), qui formule dans le délai d'un mois des propositions. Sur la base de ces propositions, le préfet peut régler le budget et le rendre exécutoire.

\section{b) L'absence d'équilibre réel du budget}

À la différence du budget de l'Etat, les budgets locaux doivent être votés en équilibre réel : les postes de dépenses ne peuvent pas être minorés et ceux des recettes ne doivent pas être arbitrairement majorés (art. L 1612 - 4 du CGCT). Par ailleurs, il faut que la dette en capital figurant en dépenses dans la section «Investissements » puisse être remboursée par des ressources définitives (hors emprunt), et certaines dépenses comme les intérêts d'emprunts ainsi que diverses autres dépenses visées par la loi doivent être obligatoirement couvertes. Si tel n'est pas le cas, le préfet doit saisir dans le mois qui suit la transmission du budget la Chambre régionale des comptes, qui dispose à son tour d'un mois pour proposer les mesures nécessaires à l'assemblée locale concernée, qui devra procéder à une nouvelle délibération. Si les nouvelles mesures votées s'avèrent insuffisantes, ou si la nouvelle délibération ne s'est pas tenue dans les délais impartis, le budget est réglé et rendu exécutoire par le préfet.

\section{c) La constatation d'un déficit après exécution du budget}

Le compte administratif établi par les gestionnaires locaux doit être voté par l'assemblée compétente avant le 30 juin de l'année qui suit celle de l'exécution du budget. En cas de déficit constaté égal ou supérieur à $10 \%$ des recettes de la section «Fonctionnement» pour les communes de moins de 20.000 habitants (5\% pour les autres), le préfet doit saisir la Chambre régionale des comptes, qui 
dispose de deux mois pour proposer les mesures nécessaires au rétablissement de l'équilibre. En outre, le budget primitif de l'exercice suivant doit aussi être transmis à la Chambre régionale des comptes par le préfet : elle lui présente alors dans le délai d'un mois, les mesures nécessaires pour remettre le budget en équilibre et que le préfet peut prendre en considération pour régler celui-ci et le rendre exécutoire. Mais le préfet peut aussi s'écarter des propositions qui lui sont faites, par un avis dûment motivé.

\section{d) Le défaut d'inscription au budget d'une dépense obligatoire}

Ce cas est plus spécifique, dans la mesure où le préfet mais aussi toutes les personnes qui y ont intérêt peuvent saisir la Chambre régionale des comptes. Celle-ci dispose d'un mois pour constater qu'une dépense obligatoire ne figure pas au budget, et adresser à la collectivité concernée une mise en demeure. Si celle-ci n'est pas suivie d'effet dans le délai d'un mois, il est demandé au préfet de procéder d'office à l'inscription de la dépense. Celui-ci n'est pas tenu d'y procéder, mais il doit alors s'en expliquer par une décision motivée de manière précise. Il peut aussi adresser lui-même une mise en demeure au gestionnaire local, lui ordonnant de mandater la dépense, et en cas de refus de sa part le préfet se substitue à lui ${ }^{60}$.

\section{C/LE CONTRÔLE DE GESTION}

Le comptable public, qui n'est pas qu'un caissier, est déjà un gardien indirect de la régularité de la gestion des organismes publics à travers ses vérifications préalables au paiement, même s'il ne peut se faire juge de la légalité des actes des ordonnateurs. Le contrôle de gestion est donc souvent lié au jugement a posteriori des comptes des collectivités locales. Mais si « les comptes font partie de la gestion, la question de la gestion ne se réduit pas à celle des comptes $»^{61}$.

Autant le jugement des comptes ne soulève pas de problèmes sérieux, car les Chambres régionales des comptes ont pu profiter de l'expérience ancienne de la Cour des comptes, autant l'examen de la gestion des collectivités locales apparaît comme une question sensible et complexe.

\section{$1 \%$ La nature et la portée du contrôle de gestion et ses implications.}

L'examen de la gestion (art. L 211-8 du Code des juridictions financières) et le contrôle de la régularité des opérations (art. L 211-3 du même Code), concernent également tous les organismes publics : collectivités locales et territoriales ainsi que leurs établissements publics en dehors de tout seuil financier, établissements, sociétés, groupements et organismes quel que soit leur statut juridique ainsi que leurs filiales, le seuil financier du contrôle étant lié à la notion de « concours

${ }^{60}$ Sur tous ces points, M. BOUVIER, Les finances locales (précité), pp. 8, 238, 242, 255 et s. ; J. MORAND-DEVILLER, op. cit., p. 237 ; G. PEISER, op. cit., p. 127 et 128.

${ }^{61}$ B. LEVALLOIS, « Quel contrôle externe des collectivités locales ? », in RFFP, n 85/2004, p. 12. 
financier $»^{62}$. Le contrôle de gestion exercé a posteriori s'intéresse notamment à la « traçabilité » des fonds publics, quel que soit le circuit emprunté

Les articles L 211-3 et L 211-8 précités du Code des juridictions financières assignent aux Chambres régionales des comptes ; d'une part un contrôle de la régularité des recettes et des dépenses et de l'emploi des crédits et des fonds, mais il porte aussi sur la régularité des actes de gestion. Cela concerne l'analyse des écritures comptables dont la Chambre régionale des comptes est le « juge » naturel. C'est pourquoi le terme "régularité » peut être entendu comme évoquant la légalité. Dès lors et dans la mesure où l'analyse de la légalité porte sur des actes qui ont engagé des fonds pour conclure des marchés publics, des conventions de délégations de services publics, ou pour attribuer des concours financiers, cela peut parfois conduire à des appréciations différentes entre le juge financier, le juge administratif, et même le juge pénal, qui pourraient tous avoir vocation à intervenir à un titre ou à un autre. D'autre part, c'est aussi la performance qui est ici examinée. Il s'agit alors un contrôle qui porte sur l'économie des moyens mis en œuvre, sur l'évaluation des résultats obtenus par rapport aux objectifs visés, mais sans pouvoir en principe remettre en cause pour autant leur opportunité. Examiner l'adéquation des moyens aux fins, cela consiste par exemple à confronter le coût d'un équipement public et les fonctions qu'il est censé remplir, ou encore à comparer le poids d'un emprunt ou d'une dette avec les capacités de remboursement. Mais dès lors que les Chambres régionales des comptes vérifient l'efficacité de la dépense publique ou la performance, cela conduit forcément à mettre en place « des indicateurs...qui permettent d'évaluer la gestion $»^{63}$.

Les Chambres régionales des comptes n'émettent normalement que des « observations » sur la gestion de la collectivité, dont les comptes sont déjà une bonne illustration. Cela ne constitue pas nécessairement une mise en cause de la gestion locale et ne débouche pas sur des procédures contentieuses, et comme il ne s'agit pas de jugements tout recours contentieux est exclu. Il est cependant possible de répondre par écrit dans le délai d'un mois aux lettres d'observations provisoires ou définitives. Formulées par le magistrat rapporteur, elles font donc l'objet d'une procédure contradictoire. S'agissant simplement de constats, leur finalité n'est pas à proprement parler de condamner, mais plutôt d'apporter une aide pour l'avenir. La loi du 5 janvier 1988 qui imposait la confidentialité des observations, a été vite supprimée par la loi du 15 janvier 1990 relative au financement des campagnes électorales. Depuis la loi du 21 décembre 2001, les élus locaux sont tenus de communiquer les rapports définitifs d'observations à l'assemblée locale pour qu'il en soit débattu. Un pas de plus a été franchi avec le décret du 3 juillet 2015, qui a procédé à de légères modifications procédurales en imposant notamment aux collectivités locales et aux établissements publics qui reçoivent un rapport d'observations définitif (ROD), de faire connaître au juge

${ }^{62}$ J.F. SESTIER, « L'examen de la gestion des collectivités locales : un contrôle nécessaire ? un contrôle accepté ? Le point de vue de l'avocat », in RFFP, $n^{\circ} 85 / 2004$, p. 68.

63 D. MIGAUD, « Nous avons des comptes à rendre », in RFFP, $n^{\circ} 85 / 2004$, p. 76. 
financier la date de la plus proche réunion de leur assemblée délibérante et de lui communiquer «en temps utile » copie de son ordre du jour. Cette publicité donnée aux observations est un élément de la démocratie locale, et c'est pourquoi les plus significatives d'entre elles figurent dans le Rapport public annuel de la Cour des comptes et portées à la connaissance des médias et de l'opinion publique. Lorsque des anomalies sont ainsi révélées par les Chambres régionales des comptes, elles doivent être accompagnées de recommandations ou de suggestions afin de les corriger ${ }^{64}$.

Si certains élus locaux supportent mal que leur gestion soit ainsi examinée et éventuellement critiquée par une institution indépendante du pouvoir politique, et s'ils sont prompts à invoquer leur légitimité élective en mettant en avant leur responsabilité politique devant l'électorat qui serait leur « seul juge », il est non moins légitime que cet électorat soit informé sur la gestion des dirigeants. Ils admettent mal aussi, de voir que les associations locales qu'ils subventionnent puissent être contrôlées et découvrir à cette occasion et à leurs dépens ce qu'est une « gestion de fait $»^{65}$.

L'examen de la gestion n'est pas une mission de nature juridictionnelle et n'a pas été conçue comme telle. Il reste que d'une part, c'est souvent à la suite de rapports d'observations que les gestionnaires locaux sont mis en cause pour gestion de fait, risquant alors d'encourir le traitement appliqué aux comptables publics passibles d'un contrôle juridictionnel. D'autre part, ce contrôle de gestion débouche in fine sur un rapport écrit de nature critique et rendu public, et la procédure qui se déroule devant une juridiction financière intègre des principes inhérents à tout procès (contradiction, collégialité, droits de la défense). Néanmoins et dans le même temps, ces observations ne sont susceptibles d'aucun recours juridictionnel. De sorte que l'on a parfois considéré que « l'examen critique de la gestion de la collectivité place la juridiction financière dans un rôle plus proche d'une Inspection générale que d'un auditeur externe $»^{66}$.

Cette question inquiète certains élus locaux qui voient là un contrôle d'opportunité, alors même que le choix des objectifs que se donnent les collectivités locales ne peut faire l'objet d'observations (loi du 21 décembre 2001 et art. L 211-8 du Code des juridictions financières). Or, il a été constaté qu' « il n'est pas rare que d'un contrôle de la qualité de la gestion, l'on passe à l'évaluation des politiques publiques locales $\|^{67}$. Mais depuis que la loi du 21 décembre 2001 a clarifié la distinction entre contrôle de régularité et contrôle d'opportunité et qu'elle

64 F. LOGEROT, « Du statut des observations des Chambres régionales des comptes », in RFFP, $\mathrm{n}^{\circ}$ 85/2004, p. 57 et s. Egalement, A. PICHON, « Le contrôle et le conseil : une cohabitation difficile », in RFFP, n 85/2004, p. 88 ; J. HOÜEL, op. cit., p. 139 ; J. P. AMOUDRY, op. cit., p. 80.

65 Elle peut être définie comme « la détention ou le maniement par une personne non habilitée de deniers qui auraient dû être encaissés et conservés par un comptable public »; cité par M. BOUVIER, Les finances locales (précité), p. 258, qui reprend une réponse ministérielle figurant au Journal Officiel, Débats-Sénat, du 20 décembre 1990. Voir également, J. MAGNET, Les gestions de fait, LGDJ, 2001

66 D. CHABANOL, op. cit., p. 55.

67 M. BOUVIER, Les finances locales (précité), p. 258. 
a amélioré les garanties de l'organisme contrôlé, il faut considérer qu'un certain équilibre a été atteint en matière de contrôle de gestion, qui reste cependant un sujet toujours controversé. Ce qui a contribué à pacifier la situation et à calmer les esprits dans une certaine mesure, a été l'instauration d'une " période trimestrielle de « silence » avant une élection locale " ${ }^{68}$. La procédure de contrôle de gestion est généralement jugée satisfaisante, grâce à ses améliorations successives et à la mise en œuvre (depuis 2013) d'un suivi des recommandations formulées dans le rôle d'audit externe indépendant attribué aux Chambres régionales des comptes. Le système est jugé adapté à la situation de la plupart des collectivités locales et des établissements publics locaux, et satisfaisant au regard des dispositions communautaires ${ }^{69}$.

\section{$2 \%$ Les problématiques inhérentes au contrôle de gestion}

L'espace local autrefois centré sur l'administration, doit aujourd'hui se confronter aux exigences de la gestion, avec les difficultés et les risques que cela implique. C'est bien pourquoi il faut en assurer le contrôle. Une culture financière nouvelle est devenue indispensable pour rationaliser la gestion, maîtriser les dépenses publiques. Tant pour l'Etat que pour les collectivités locales, c'est la logique de performance qui doit être privilégiée dans leur gestion, dont la professionnalisation a pu progresser. C'est là une exigence démocratique nouvelle, surajoutée à la composante juridique et politique traditionnelle qui a contribué à la clarification et à la meilleure transparence des comptes publics.

Au cours des dernières décennies, on a donc vu cohabiter deux cultures : l'une plus classique découlant du droit dans sa dimension parlementaire et démocratique, et l'autre liée à une conception plus économique de l'action publique valorisant la gestion. C'est donc la recherche de l'efficacité optimale qui est au centre du droit public financier actuel. Celui-ci doit concilier la tradition juridique et la modernité gestionnaire. C'est pourquoi, « outre les problèmes concernant le financement des collectivités locales et leurs relations avec l'Etat $\aleph^{70}$, il faut faire cohabiter le contrôle de régularité et l'examen de la gestion impliquant l'évaluation de la performance. Il s'agit d'éviter le décalage entre les règles nées dans un contexte longtemps figé de dépendance des collectivités locales envers l'Etat, et les impératifs actuels privilégiant l'efficacité d'une gestion confrontée à la complexité. Dans ces conditions, le contrôle de régularité adapté à une situation où les initiatives locales sont réduites, n'est plus approprié au dynamisme exigé de l'action locale. Il peut même constituer une cause de blocage d'une gestion locale, qui, y compris dans sa composante financière, relève plutôt d'un contrôle de gestion inspiré du management privé. Pour l'observateur avisé qu'est M.

${ }^{68}$ A. PICHON, op.cit., p. 88. Egalement, A. LAMBERT, op. cit., p. 92 et D. MIGAUD, « Nous avons des comptes à rendre » (précité) p. 75.

${ }_{69}$ Un plus haut niveau d'exigences pourrait concerner les très grandes collectivités et les grands établissements publics de coopération intercommunale spécialisés; selon F. ADVIELLE et P. VAN HERZELE, «Vers une assurance renforcée ... », (précité), p. 560.

70 M. BOUVIER, Les finances locales (précité), p. 231. 
BOUVIER, cela a conduit « à la rencontre de deux types de contrôle : le contrôlevérification qui répond à un objectif politique et juridique, et le contrôle-régulation qui répond à un objectif de gestion $\rangle^{71}$. Le risque actuel dans le contrôle de la gestion des collectivités locales désormais privilégié, serait cependant de négliger les contraintes juridiques, au nom des impératifs de rapidité dans l'action et du recours nécessaire à des techniques sophistiquées pour réaliser certains projets. Le droit ne doit pas être conçu ou perçu comme un obstacle à la gestion performante, et les collectivités publiques ne sont pas des entreprises, dont le modèle ne saurait être transposé sans adaptation adéquate.

Le droit public ne doit pas être un frein à la modernisation de la gestion étatique ou locale, et les finances publiques ne doivent pas devenir une zone de non-droit. Il y va de la sécurité des rapports, aussi bien au sein des Etats qu'entre eux : c'est le danger que ferait planer au plan interne comme au niveau interétatique le primat de la réalité économique et gestionnaire sur le droit. Sans sombrer dans un juridisme étroit et sans opposer les juristes aux gestionnaires, il s'agit d'intégrer la régulation de la décision (y compris son exécution) ainsi que de la gestion, dans un système associant un contrôle interne fonctionnant de manière continue et un contrôle externe indépendant vérifiant la régularité des opérations.

\section{III - L'ACTIVITÉ DES CHAMBRES RÉGIONALES ET TERRITORIALES DES COMPTES : DE LA CULTURE DES PROCÉDURES À CELLE DES RÉSULTATS}

Les cadres de la vie locale se sont beaucoup modifiés ces dernières années et aussi diversifiés, avec la multiplication des satellites des collectivités locales et de leurs groupements. Une nouvelle logique de gestion et de contrôle s'est également développée (notamment avec la LOLF). Face à ces transformations, les CRTC ont ainsi fait évoluer le contrôle traditionnel portant sur la régularité des décisions financières, pour aboutir à l'évaluation de la performance. Par ses méthodes d'enquête, sa position institutionnelle, son expertise, la juridiction financière dans son ensemble, dont le champ de compétence est large, est apte à rendre compte d'une action publique qui associe aujourd'hui de nombreux intervenants. En s'adaptant aux spécificités de la nouvelle gestion locale, grâce aux enquêtes communes à plusieurs CRTC auxquelles la Cour des comptes peut être associée, elles sont en mesure d'évaluer les politiques partenariales complexes, d' « identifier les acteurs ou les facteurs auxquels sont imputables les résultats observés $»,{ }^{72}$ et de livrer un diagnostic global déterminant la contribution de chaque intervenant.

71 Eod. loc., p. 232.

72 D. LAMARQUE, « L'évaluation des politiques publiques locales par les chambres régionales des comptes : jusqu'où », in RFFP, n 85/2004, p. 63. 


\section{§ 1. L'état des lieux en matière de gestion locale}

Les mutations intervenues dans le paysage local et en matière de culture financière ont été entièrement prises en compte et assumées par les CRTC.

\section{A/ LES CONSTATS GÉNÉRAUX EN MATIÈRE D'INFORMATION FINANCIÈRE}

Il est essentiel pour le juge financier de prendre la mesure non seulement des recettes et des dépenses, mais aussi des engagements et des risques financiers pris par les collectivités locales notamment dans les organismes extérieurs. À la suite de la modification des seuils de contrôle ${ }^{73}$, les CRTC ont été conduites à exercer leur mission sur 3606 communes au lieu de 9354 précédemment, sur 1545 établissements publics de coopération intercommunale au lieu de 2763 auparavant et sur 312 établissements publics d'enseignement au lieu de 8128 antérieurement ${ }^{74}$. Mais les comptes et la gestion des petites communes peuvent toujours être contrôlées si la situation l'exige.

Ce contrôle peut d'autant plus facilement s'exercer que les informations financières présentées sont complètes, fiables et vérifiables dans des délais raisonnables. Or, dans les années qui ont suivi la décentralisation, il s'est avéré que les services communaux ne transmettaient pas au comptable toutes les factures, ce qui conduisait à minorer fictivement les dépenses ${ }^{75}$. En outre, des communes pouvaient avoir mal négocié certains contrats, ou avoir accordé leur garantie d'emprunt ou s'être liées à des organismes locaux, ou encore s'être exposées à des contentieux pouvant s'avérer coûteux. À ces risques d'ordre financier pouvaient s'en ajouter d'autres liés à des retournements de conjoncture ou à des situations objectives (communes littorales victimes de pollutions maritimes, communes touristiques subissant des intempéries, ou stations de montagne privées d'enneigement). Les déboires du principal établissement de financement des collectivités locales (DEXIA), qui a été frappé par la récente crise financière, a prouvé à quel point la trop grande liberté d'emprunter ou de placements dans des produits financiers pouvait mettre en péril les finances locales et par contrecoup celles de l'Etat ${ }^{76} \mathrm{~A}$ côté des opérations banales des collectivités locales, il y en a d'autres qui le sont moins, comme les délégations de services publics ou les investissements à risques, qui appellent un contrôle plus poussé.

73 Loi du 13 décembre 2011 qui confie aux directeurs départementaux ou régionaux des finances publiques le soin de procéder à l'apurement administratif des comptes des collectivités locales situées sous les seuils démographiques et financiers (Voir Supra). Sur ce point : G. MILLER et F. ADVIELLE, op. cit., p. 591.

74 Chiffres cités par S. DAMAREY, op. cit., p. 320. D'autres chiffres sont rapportés in M. BOUVIER, Les finances locales (précité), p. 255, où il est fait état de 3012 jugements en 2012, 659 avis de contrôle budgétaire et 620 rapports d'observations définitives.

75 J. HOUEL, op. cit., p. 144.

76 Voir le Rapport sur « La gestion de la dette publique locale », La Documentation Française, juillet 2011, pp. 93 et 94. Egalement, M. CONAN, « L'autonomie financière des collectivités territoriales : trente ans après la loi de décentralisation du 2 mars 1982, état des lieux », in A.J.D.A., n 14/2012, p. 762. 
Le contrôle exercé par les CRTC révèlent encore des lacunes dans la tenue des comptabilités locales dont la fiabilité peut être problématique ; soit que les engagements pris ne sont pas complètement recensés, soit que le passif exact est mal connu, soit que les collectivités locales ont une connaissance imparfaite de leur patrimoine. Ces constats permettent de dire que « la situation actuelle de l'information financière locale ne peut être considérée comme pleinement satisfaisante malgré les nombreux progrès enregistrés depuis vingt ans $»^{77}$. Il ne s'agit plus seulement d'informer les citoyens contribuables et les usagers des services publics, qui tous attendent des prestations adaptées à leurs besoins et au meilleur rapport qualité/prix, mais encore on voit se développer des systèmes de notation des collectivités locales les mieux gérées, similaires au «rating » du secteur privé. Pour parvenir à la meilleure utilisation possible des ressources locales, un effort doit être fait déjà au stade du contrôle interne, avant qu'interviennent les divers contrôles externes.

Quant aux suites données aux observations définitives des juridictions financières en général, la loi du 13 décembre 2013 a prévu que le rapport public annuel de la Cour des comptes en comportera une présentation.

\section{B/ LES CONSTATS THÉMATIQUES}

Les CRTC ont développé des enquêtes communes en matière d'assainissement, de voirie, de gestion des déchets ménagers, de transports urbains, de distribution d'eau, de politique de la ville, de gestion du système éducatif, de gestion hospitalière, entre autres. Les juridictions financières ont prouvé leur capacité à « examiner ensemble les politiques publiques transversales qui associent l'Etat et les collectivités locales ${ }^{78}$.

Du côté des gestionnaires locaux, de nombreuses actions correspondent à des projets complexes caractérisées par des objectifs multiples ${ }^{79}$, ou conduites en partenariat avec des formes plus ou moins bien définies et une répartition des compétences insuffisamment nette. Parfois c'est le cloisonnement dans l'action ou le manque de concertation des acteurs locaux, ou l'octroi de soutien financier insuffisant ou excessif qui ont pu faire l'objet de recommandations, d'ailleurs non toujours suivies d'effets ${ }^{80}$. Quelques exemples seront ici mentionnés.

77 F. ADVIEILLE et P. VAN HERZELE, « Vers une assurance renforcée... » (précité) p. 560

78 F. LOGEROT, op. cit.,

79 La politique de la ville en est un très bon exemple, qui touche à l'urbanisme, à l'habitat, mais aussi à l'éducation, au domaine social et même au-delà.

80 D. MIGAUD, en a donné quelques exemples éloquents lors de la présentation du Rapport Public 2015 de la Cour des comptes, in « La performance de 1'action publique », (précité) p. 6.Il cite notamment les cas suivants :

- l'affaire des aéroports de Dole et de Dijon, dont le développement n'a pas été concerté, malgré les recommandations de la CRC de Bourgogne-Franche-Comté, dont le bilan financier est catastrophique, impliquant un soutien financier sans rapport avec le trafic aéroportuaire, le département du Jura ayant versé des aides non notifiées à la Commission européenne, qui, si elles sont jugées incompatibles avec les Traités européens, devront être remboursées ;

- l'exemple du Musée des civilisations de l'Europe et de la méditerranée à Marseille qui a ouvert en 


\section{$1 \%$ Les mutualisations des services et les intercommunalités.}

La mutualisation des services permet de concilier l'attachement au territoire et à ses particularités, avec les potentialités qu'offre la coopération entre collectivités locales. Ces dernières sont appelées avec de plus en plus d'insistance à participer à la contrainte financière, afin de faciliter le retour des comptes publics à l'équilibre, en prenant en considération les impératifs communautaires. Les juridictions financières ont notamment suivi de près les évolutions des dépenses en personnels dans la fonction publique locale ${ }^{81}$. Il en résulte un coût élevé pour les contribuables, sans supplément d'efficacité notable pour l'action publique. Tout en faisant preuve de prudence dans leurs observations, les chambres régionales des comptes encouragent cependant la mutualisation ${ }^{82}$. Parmi les dysfonctionnements signalés, elles relèvent l'insuffisante coordination des acteurs publics locaux, le manque de clarté dans la répartition des compétences, un partage des coûts non conforme à la réalité. Le manque de précision des conventions de mutualisation, qui nuit à la bonne compréhension des obligations respectives conduit à recommander une évaluation des résultats de ces conventions au terme de leur première année d'application ${ }^{83}$.

La mutualisation des services et la péréquation des ressources sont au fondement de l'intercommunalité, qui permet aux collectivités locales d'offrir des services et une qualité de prestations dont elles ne peuvent pas supporter le coût isolément. L'intercommunalité vise à constituer dans les zones aussi bien rurales qu'urbaines, des ensembles territoriaux cohérents organisés en établissements publics de coopération intercommunale (EPCI), avec ou sans fiscalité propre. La mise en commun de moyens financiers et en personnels permet aux communes partenaires de réaliser des projets dépassant les possibilités de chaque commune membre prise individuellement. Dans leur évaluation de ces groupements, les juges financiers ont été conduits à constater parfois l'inadaptation du périmètre territorial de certains d'entre eux, le caractère imprécis ou l'exercice incomplet des compétences transférées, la définition trop vague de l'intérêt communautaire,

2013 au lieu de 2008 et coûté plus de 310 millions d'euros, est un autre exemple de gestion défaillante dans la mesure où les frais de fonctionnement sont excessifs au regard des ressources disponibles ;

- le financement et le développement des stations de skis pyrénéennes n'ont pas pris en compte les mutations possibles ni les projets identiques projetés du côté espagnol, obérant la rentabilité des opérations et perturbant ainsi l'économie locale ;

- l'absence de coordination en matière de transports urbains de voyageurs a aussi été mentionnée, dont le développement n'est pas assez coordonné alors que le poids financier s'accroît avec l'allongement des réseaux et les coûts en personnels.

81 F. ADVIELLE, « Les chambres régionales et territoriales des comptes et les mutualisations des services » in A.J.D.A., $n^{\circ}$ 35/2012, p. 1952 : l'auteur relève que l'Observatoire des finances locales a noté une progression des dépenses de personnels de 44,8 \% entre 2004 et 2012, soit pour un montant de 58 milliards d'euros, représentant 35,7\% des dépenses de fonctionnement contre $34 \%$ en 2004.

82 CRC Pays de Loire, 28 novembre 2011, Communauté de Communes Castelbriandais : CRC, 3 août 2011, Communauté d'agglomération de Limoges métropole. Sur ce point, F. ADVIELLE, eod. loc., p. 1595.

83 CRC Lorraine, 6 octobre 2010, Commune de Pompey 
Marc Gjidara: Le contrôle de la gestion locale par les chambres régionales et territoriales des comptes Zbornik radova Pravnog fakulteta u Splitu, god. 53, 1/2016., str. 67.-103.

certaines intercommunalités exerçant même des compétences qui ne leur sont pas formellement attribuées ${ }^{84}$

\section{$2 \%$ Les activités externalisées des collectivités locales}

L'appel à des opérateurs externes est une possibilité largement utilisée par les collectivités locales. Elles ont alors recours notamment à des associations déjà existantes ou créées ad hoc, auxquelles sont attribuées des subventions ou confiés des moyens matériels ou des équipements. Les divers modes de gestion externalisée sont validés, mais assortis de critères de légalité dont le respect est parfois difficile à apprécier. Le Conseil d'Etat a récapitulé les différentes formules contractuelles utilisables par les collectivités locales, pour confier à un opérateur extérieur une activité constituant une activité de service public, ou pour orienter l'activité d'un organisme privé sans pour autant faire de cette activité un service public $^{85}$. Seuls certains services sont insusceptibles d'être ainsi délégués en raison de leur nature particulière ; c'est le cas de l'organisation et du contrôle du stationnement payant ou de la surveillance des élèves durant les pauses dans la vie scolaire. La loi du 12 avril 2000 (art. 20) relative aux droits des citoyens dans leurs relations avec les administrations, impose la conclusion d'une convention avec les opérateurs privés bénéficiaires de subventions ${ }^{86}$, définissant leur objet, leur montant et les conditions de leur utilisation.

Dans ce domaine, les juges financiers sont souvent conduits à « se prononcer sur la nature juridique réelle des situations...et parfois ne peuvent se satisfaire des appellations plus ou moins exactes et précises des documents et actes examinés et les requalifient avant d'en tirer les conséquences quant à la régularité des décisions et des opérations $»^{87}$. Cet examen peut notamment aboutir à la requalification en service public de l'activité subventionnée. La requalification peut aussi révéler dans certains cas un manquement aux règles de mise en concurrence, ce qui peut donner lieu à des poursuites pour délit de favoritisme (art. 432-4 du Code pénal). L'arrêt du Conseil d'Etat, Commune d'Aix-en-Provence (précité) concerne les cas où une collectivité locale peut confier par contrat la gestion d'un service public à un tiers, sans recourir à une délégation de service public ou à un marché public sans mise en concurrence ${ }^{88}$. Cela peut se justifier si le lien avec le tiers correspond à une prestation intégrée dite " in house », qui est strictement interprétée par la jurisprudence administrative et de ce fait rarement retenue par le juge financier. L'autre situation est celle où le lien contractuel vise à organiser ou à financer une activité présentant un caractère d'intérêt général prédominant. En dehors de

\footnotetext{
84 G. MILLER et A. LEYAT, « L'intercommunalité », in A.J.D.A., n 13/2009, p. 699 et s.

85 C.E., Section, 6 avril 2007, Commune d'Aix-en-Provence.

${ }^{86}$ Lorsque la subvention dépasse un certain montant fixé par décret (seuil fixé à 23.000 euros par le décret du 6 juin 2001)

${ }^{87}$ F. ADVIELLE et P. VAN HERZELE, « Les Chambres régionales des comptes et les activités externalisées des collectivités locales », in A.J.D.A., n 35/2013, p. 2022.

88 Ce régime dérogatoire s'applique lorsque l'organisme tiers n'est pas « un opérateur sur un marché concurrentiel ».
} 
ces cas-là, toute délégation de service public et tout marché public impliquent une publicité et une mise en concurrence adéquates. Les chambres régionales des comptes font souvent des observations sur ce point et procèdent s'il le faut à la requalification du lien existant entre la collectivité locale et son opérateur.

L'éventuelle requalification découle de l'examen cas par cas de chaque activité externalisée, non seulement dans le but de restituer la qualification juridique exacte de l'opération, mais aussi pour parer aux implications économiques et aux risques financiers éventuellement encourus par la collectivité publique. Le contrôle du juge financier porte aussi sur la façon dont l'opérateur a été suivi, sur l'équilibre financier et sur la maîtrise de l'activité qui lui est confiée. Car les collectivités locales, même disposant de services compétents, éprouvent des difficultés à faire respecter par leurs opérateurs les obligations qui leur incombent. La pratique a montré que bien souvent une part croissante du coût des prestations assurées par les opérateurs est reportée sur les collectivités locales, et que dans certains cas ce sont elles qui supportent des risques financiers, en dépit des textes qui visent à limiter leur exposition à de tels risques.

La complexité des situations rencontrées justifie la variété et le nombre des observations émanant des juges financiers, qui doivent exercer une vigilance particulière sur le degré de précision avec lequel les collectivités locales définissent, notamment en matière d'encaissement des recettes, les obligations pesant sur les opérateurs et les organismes aidés matériellement ou financièrement dans l'exercice des activités d'intérêt général qui leur sont confiées. L'examen de la gestion par les chambres régionales des comptes (art. L 211-8 du Code des juridictions financières) s'exerce en vue de garantir aussi bien la sécurité juridique des opérations que les intérêts financiers des collectivités publiques. Ce contrôle englobe la régularité des actes de gestion, l'économie des moyens engagés et l'évaluation des résultats atteints par l'organisme concerné par rapport aux objectifs qu'il s'est fixés.

Pour toutes ces raisons, «l'examen des activités externalisées des collectivités locales est appelé à mobiliser durablement les chambres régionales des comptes $»^{89}$

\section{3\% Le Sociétés d'économie mixte locales}

Dans la mesure où il s'agit d'organismes servant à prolonger l'action des collectivités locales, ceux-ci se sont révélés adaptés à leur mission dans divers secteurs d'activité ${ }^{90} \mathrm{Si}$ leur nombre a légèrement diminué, on en comptait encore environ 1.100 en 2008, représentant 2,8 milliards d'euros en capital dont $65 \%$ 2088.

${ }^{89}$ F. ADVIELLE et P. VAN HERZELE, « Les CRC et les activités externalisées... » (précité), p.

90 Les sociétés d'économie mixte interviennent notamment dans le secteur de la construction et la gestion de logements, les transports publics, les parcs de stationnement, les transports publics, la production et la distribution d'eau, les réseaux d'énergie ou de chaleur, la gestion d'équipements, la promotion et la commercialisation touristiques, les ports de plaisance, la gestion des ports et aéroports. Depuis quelque temps leurs activités se sont étendues au secteur de la santé et s'exercent toujours en matière d'aménagement. 
Marc Gjidara: Le contrôle de la gestion locale par les chambres régionales et territoriales des comptes Zbornik radova Pravnog fakulteta u Splitu, god. 53, 1/2016., str. 67.-103.

détenus par les collectivités locales, alors que leur chiffre d'affaires dépassait à l'époque les 10 milliards d'euros ${ }^{91}$. Leur importance exige donc un contrôle vigilant qui est la garantie démocratique de l'usage conforme et efficace de l'argent public. Bien que fonctionnant sous le régime juridique applicable aux sociétés commerciales, les sociétés d'économie mixte locales sont soumises au contrôle exercé par les chambres régionales des comptes sur leurs comptes et leur gestion.

C'est ainsi que les observations des CRTC concernent tout d'abord la fiabilité des comptes, et qu'il en ressort que les actionnaires publics ne sont pas toujours en mesure d'exercer leur contrôle. Parfois les collectivités locales ne sont pas en situation de vérifier la bonne exécution du service qui a été délégué et de recueillir l'information qu'elles doivent aux citoyens ${ }^{92}$. Des anomalies sont aussi relevées dans l'attribution ou le renouvellement des délégations de service public, et parfois les modalités de la délégation ont pour effet de reporter sur le budget de la collectivité locale les risques d'exploitation. Il est souvent rappelé par le juge financier, que la loi du 29 janvier 1993 relative à la prévention de la corruption et à la transparence de la vie économique et des procédures publiques, impose de respecter les règles de publicité et de mise en concurrence prévues par le Code des marchés publics et que cela vaut pour les contrats de travaux, d'études et de maîtrise d'œuvre, conclus par les sociétés d'économie mixte pour l'exécution ou les besoins du service public, que ce soit en leur nom ou pour le compte de personnes publiques ${ }^{93}$

Parmi les autres anomalies, figurent les aides directes ou indirecte accordées irrégulièrement aux sociétés d'économie mixte par les collectivités publiques actionnaires $^{94}$. La gestion des personnels de ces sociétés d'économie mixte locales, présente aussi de nombreuses déficiences liées à l'augmentation excessive des dépenses réalisées à ce titre ${ }^{95}$. En matière d'aménagement, certains projets

91 G. MILLER et A. LEYAT « Les chambres régionales des comptes et le contrôle des sociétés d'économie mixte locales », in A.J.D.A., n 31/2009, p. 170 et s.

${ }_{92}$ CRC Ile de France, 8 mars 2006, Société Nouvelle d'exploitation de la Tour Eiffel.

93 C.R.C. Alsace, 14 septembre 2004, Compagnie des transports strasbourgeois.

94 G. MILLER et A. LEYAT, « Les CRC et le contrôle des sociétés d'économie mixte locales » (précité), p. 1.704 ; mentionnant des cas d'aides plus ou moins masquées, comme celui, où tous les marchés de communication d'une ville actionnaire d'une société d'économie mixte sont attribués à celleci sans mise en concurrence véritable, les prestations étant par ailleurs surpayées, ce qui permettait à la même société de financer ses autres activités potentiellement déficitaires (C.R.C. Provence-Alpes-Côte d'Azur, 22 mars 2004, Société d'économie mixte Martigues Communication). Un autre cas est celui de l'achat de locaux par une société, suivi de leur revente à son actionnaire public majoritaire 4 ans après avec une plus-value de $65 \%$, ce qui équivaut à une aide indirecte (C.R.C. Champagne-Ardenne, 30 mars 2004, Société d'économie mixte d'Epernay et de sa région). Il en va de même quand une collectivité locale actionnaire d'une société d'économie mixte s'abstient de lui faire payer une redevance pour la location d'équipements (C.R.C. Bourgogne, 23 décembre 2004, SAEMS du circuit de Nevers-Magny-Cours).

95 Notamment à cause du montant exagéré des primes et indemnités, du laxisme observé dans le remboursement des frais professionnels. Sont également incriminés le manque de maîtrise des frais généraux, des pratiques parfois dispendieuses en matière de communication, l'opacité et la croissante anormale des frais de carburant des véhicules de service dont l'utilisation n'est pas règlementée, la gestion anormale des marchés, avec recours abusif aux marchés négociés sans mise en concurrence préalable. Sur 
trop ambitieux sont parfois engagés alors que leur viabilité économique n'est pas envisageable. Il arrive également que les représentants de la collectivité locale au sein du conseil d'administration ne tiennent pas informée l'assemblée locale concernée, ou le font trop tard ou de manière incorrecte, en violation des dispositions légales, quand ce n'est pas la transmission des comptes qui est omise ou tardive..$^{96}$

Tous ces exemples illustrent la tendance du juge financier à aller au-delà de la seule appréciation de la régularité formelle de la gestion et de la situation financière, pour s'orienter vers l'évaluation.

\section{4\% L'activité des régions}

Avec les réformes successives, les compétences des régions se sont notablement accrues et la structure de leurs ressources a évolué avec le renforcement de la fiscalité locale. Alors que les collectivités locales, y compris les régions, doivent contribuer aux efforts financiers pour permettre un retour à l'équilibre des comptes publics, les juges financiers confrontés à des nouveaux modes de gestion se sont montrés de plus en plus vigilants.

L'action des régions s'est accentuée dans plusieurs domaines, dont le développement économique, les grandes infrastructures (ports et aéroports), l'aménagement du territoire, la politique culturelle, la formation professionnelle continue et l'apprentissage ainsi qu'en matière d'éducation ${ }^{97}$ S'agissant de la gestion des personnels, et comme pour les autres collectivités locales contrôlées, les chambres régionales des comptes ont constaté une augmentation excessive des indemnités, un laxisme coupable dans la lutte contre l'absentéisme au travail, un non respect patent de la durée du temps de travail, des recrutements injustifiés (sans nouvelles attributions de compétences aux régions) et parfois même irréguliers (notamment d'agents contractuels de direction et d'encadrement). Si les juges financiers ont pu renouveler leurs analyses, amélioré leur connaissance du patrimoine des régions et de leurs établissements publics, il leur a aussi été donné de constater que " la comptabilisation des opérations complexes reste encore aléatoire voire peu protectrice des intérêts de la collectivité $\rangle^{98}$. Dans leurs observations, ils soulignent « les limites de la fiabilité des comptes présentés à l'assemblée délibérante $\gg^{99}$ Les recommandations insistent sur la nécessaire clarification des attributions des régions et de la répartition des compétences entre

tous ces points, G. MILLER et A. LEYAT, Eod. loc., p. 1705.

96 En méconnaissance de l'article L 1524 -5 du CGCT À titre d'exemples : C.R.C. Ile de France, 22 novembre 2004, SODAME ; C.R.C. Haute-Normandie, 4 octobre 2004, SEM du Golf à Evreux ; C.R.C. Languedoc-Roussillon, 31 janvier 2007, S.A. Elit ; C.R.C. Aquitaine, 22 juin 2007, SEMITOUR-Périgord.

97 Les régions ont la responsabilité des lycées et de certains établissements spécialisés, pour la construction, l'équipement et le fonctionnement, y compris le recrutement et la gestion des personnels non enseignants.

98 Sur tous ces points, F. ADVIELLE et P. VAN HERZELLE, « Les chambres régionales et territoriales des comptes et la situation des régions », in AJDA, n 10/2013, p. 572.

99 Ibidem 
acteurs locaux, dont les actions doivent être mieux coordonnées. Les politiques conduites au plan régional doivent également faire l'objet d'évaluations plus précises.

\section{§ 2. La nouvelle gouvernance locale}

Aujourd'hui, les collectivités locales doivent pouvoir évaluer l'évolution de leur gestion dans le temps et se comparer dans l'espace aux autres types de gestion, pour identifier les bonnes pratiques et faire un usage optimal de leurs ressources.

\section{A/ D'UNE ADMINISTRATION DE PROCÉDURES À UNE ADMINISRATION DE RESPONSABILITÉ}

La responsabilisation des collectivités locales et de leurs gestionnaires a commencé à évoluer dans les années 1970. Ce processus a connu plusieurs étapes, avec tout d'abord la reconnaissance d'un début d'autonomie de décision en matière financière ${ }^{100}$, suivie de l'octroi d'une autonomie fiscale limitée au vote des taux des principaux impôts directs locaux par les assemblées locales. Enfin, la proclamation du principe de subsidiarité a conduit à une augmentation des actions locales, et corollairement à un accroissement des budgets locaux et de leurs objectifs. Avec les crises financières à répétition, il a cependant fallu gérer plus rationnellement les finances publiques, ce qui a conduit à une plus grande responsabilisation des gestionnaires publics y compris au niveau local. Il est ainsi devenu nécessaire d'adopter une conception plus intégrée des finances publiques avec toutes les conséquences qui en découlent, tout en préservant le principe de libre administration locale. Le but a été de «mettre en cohérence le système financier public en dégageant une logique commune d'évolution des dépenses et recettes $»^{101}$. Cela impliquait une certaine normalisation de la gestion locale engagée par la loi du 6 février 1992 qui a imposé une amorce de consolidation des comptes. Puis la loi du 22 juin 1994 a réformé la comptabilité communale en la rapprochant de celle des entreprises, ouvrant ainsi la voie à une nouvelle gestion des finances publiques. Cet éclairage nouveau destiné à modifier l'action des pouvoirs financiers locaux devait permettre d'améliorer la qualité des comptes. Cela a résulté de la normalisation comptable réalisée par l'harmonisation de la comptabilité publique avec le Plan comptable général en vigueur dans le secteur privé. Ce processus a été parachevé avec la loi organique relative aux lois de finances (LOLF), qui a visé à moderniser la gestion publique dans son ensemble en la faisant passer « d'une administration de procédures à une administration de

100 Avec le système de la globalisation des prêts par les différents organismes financiers (Caisse des dépôts et consignations, Caisses d'épargne, Caisse d'aide à l'équipement des collectivités locales).

101 M. BOUVIER, Les finances locales (précité), p. 42. 
responsabilité $»^{102} \mathrm{Ce}$ texte fondamental relatif aux finances de l'Etat réalisait une réforme de la gestion publique en plus d'une réforme budgétaire ${ }^{103}$.

La nouvelle logique de responsabilité fondée sur une culture du résultat, a représenté un saut qualitatif conduisant les collectivités locales à revoir leurs techniques et leurs procédures.

\section{B/ LA QUESTION DE LA PERFORMANCE DANS LA GESTION LOCALE}

La loi organique relative aux lois de finances adoptée en 2001 a aussi fait de la performance un critère primordial de la bonne gestion, tout en assortissant la liberté d'action d'un contrôle a posteriori, qui a ajouté à l'objectif de la régularité une préoccupation d'efficacité de la gestion financière.

Dans le système ancien, l'important était de s'en tenir à la réglementation en respectant le montant des dépenses autorisées, la responsabilité des gestionnaires étant appréciée en fonction de l'application des règles et non pas des résultats obtenus. Or, le budget de résultats substitué au budget de moyens met l'accent sur la performance de la dépense publique. C'est par rapport aux résultats à atteindre qu'est jaugée la responsabilité managériale vis-à-vis des citoyens, des contribuables, des usagers. Ce système implique " de produire des résultats chiffrés fiables et non biaisés (et) des commentaires sur les écarts entre les cibles de résultat et les résultats atteints (qui soient) exhaustifs et sincères $»^{104}$.

C'est dans ce contexte et à la faveur de la décentralisation que les collectivités locales ont adopté des méthodes de gestion inspirées du management privé. L'Etat se révélant incapable de stabiliser l'économie, l'attitude consistant à partager la richesse a cédé la place à l'idée de répartition des efforts sur tous les gestionnaires publics. La culture financière publique a intégré l'impératif d'une gestion plus rationnelle de l'argent public. C'est ainsi que les collectivités locales, placées à l'interface du secteur public et du secteur privé et stimulées par l'élargissement de leurs compétences, ont adopté une nouvelle gouvernance, ni exclusivement publique, ni véritablement privée. Les différences avec les entreprises privées subsistent, car « dans un cas le marché régulateur suprême sanctionne en temps réel l'entreprise par rapport à son objectif qu'est le profit. Dans l'autre cas, les objectifs fondamentaux sont souvent ambigus et contradictoires $»^{105}$. En effet, il s'agit pour les gestionnaires locaux de répondre aux besoins de la collectivité, d'ordre économique, social et politique. C'est pourquoi la gestion locale s'est progressivement professionnalisée, mettant parfois en place un contrôle de gestion voire d'évaluation ${ }^{106}$, qui tend à vérifier l'adéquation réalisée ou non entre les

${ }^{102}$ S. FLIZOT, Les responsabilité financière des gestionnaires publics en Europe » 'précité), p. 709.

103 En organisant « la présentation, la discussion et le vote du budget selon un nouveau cadre autour des politiques publiques $\gg$.

104 A. BARILARI, op. cit., p. 700.

105 E. PORTAL, op.cit., p. 111.

106 Cette évolution a été encouragée dès 1988, s'agissant de l'évaluation obligatoire des fonds structurels : la politique de la ville et l'action économique ont participé aux actions contractualisées 
besoins identifiés et les résultats des actions entreprises ${ }^{107}$. Mais cette évaluation n'est pas toujours facile, en particulier à propos des politiques partenariales, car il faut apprécier les résultats par rapport à des critères qui peuvent varier en fonction du niveau auquel les actions ont été conduites ${ }^{108}$.

L'évaluation ne peut reposer que sur un système d'informations approprié permettant de mesurer les résultats, implique une culture partagée et un partenariat entre contrôle interne et contrôle externe. C'est ce qui permet de juger de la performance et des effets de l'action publique. À cet égard, les chambres régionales des comptes insistent sur le fait que la définition des objectifs doit être précédée de l'analyse des besoins, et que la comparaison entre les collectivités locales suppose une information homogène, fiable, cohérente et axée sur la performance. Cette notion de performance est indissociable de l'évaluation qui apprécie si les objectifs sont atteints, s'ils l'ont été avec toute l'efficacité souhaitable et au meilleur coût.

Comme les citoyens sont devenus de plus en plus exigeants en matière d'efficacité et de qualité des prestations offertes par les services publics, les collectivités locales, avant l'Etat lui-même, ont inscrit leur gestion dans une culture de performance et d'évaluation pour répondre à la demande d'information sur leur gestion. Cette attitude de leur part s'inscrit dans le droit fil de la loi organique sur les lois de finances (LOLF) qui a promu la culture de la performance désormais substituée à la tradition administrative caractérisée par la priorité donnée au droit. La norme se trouverait ainsi relativisée par rapport à la performance, dont les critères sont multiples et surtout économiques et sociaux.

Tout semble donc opposer le droit et la performance. En effet, « fondée sur un référentiel juridique la norme a le caractère d'un principe général, elle appelle un contrôle de régularité débouchant sur la sanction $»^{109}$. En réalité, le droit et le management ne sauraient se contredire et ils s'informent réciproquement, car les notions d'efficience et d'efficacité de la gestion sont déjà intégrées au monde du droit, notamment en jurisprudence ${ }^{110}$. Confirmant que le droit et la performance ne sont pas des notions tout à fait étrangères l'une à l'autre, J. CAILLOSSE a pu écrire, que « le droit ne peut rester insensible au désir grandissant de performances

qui ont conduit à un grand nombre d'évaluations. En 2004, les deux tiers des villes de plus de 50.000 habitants avaient adopté des démarches d'évaluation volontaire ou obligatoire. Sur tous ses points, D. LAMARQUE, op. cit., p. 62.

107 Cela doit être fait au plus près du terrain, car c'est à ce niveau que l'on peut évaluer les résultats.

108 La politique des transports en est un exemple : elle n'a pas le même sens au niveau national (où il s'agit de promouvoir un aménagement harmonieux du territoire) ou au niveau européen (visant à faciliter la circulation des personnes et des biens). Il en va de même entre le niveau régional où l'on vise le développement économique en interconnectant des réseaux, et le niveau infra régional où l'on cherche à développer l'emploi et à préserver l'environnement.

109 D. LAMARQUE, op. cit., p. 60.

110 Le contrôle de proportionnalité en est un bon exemple, puisque cela consiste à apprécier le bien fondé d'une réglementation par rapport au contexte et aux circonstances concrètes, en prenant en compte notamment l'équilibre entre les avantages d'une opération ou d'une décision et les inconvénients qui en découlent. 
publiques (et) ces dernières ne peuvent se passer d'une expression juridique $»^{111}$.

À cette évolution de la gestion publique et de la responsabilité des gestionnaires, correspond du côté des juges financiers l'émergence et l'affirmation de solutions novatrices dictées par un nouveau contexte.

\section{CONCLUSION}

À côté du juge administratif, le juge financier est un acteur différent, mais tous deux sont au service de la même cause : l'utilisation conforme et optimale des ressources publiques au service des citoyens.

Si en 2007 le chef de l'Etat voulait faire de la juridiction financière « le grand organisme d'audit et d'évaluation dont (l') Etat a besoin ${ }^{112}$, sa mission ne peut-être réduite à ce seul rôle. Les quatre métiers de la juridiction financière sont toujours: l'information des citoyens, la certification des comptes publics, l'évaluation des politiques publiques, la responsabilisation des gestionnaires publics ${ }^{113}$. Le contrôle exercé par une juridiction de l'Etat sur les comptes et la gestion des collectivités locales est légitime, et son indépendance permet de garantir que les comptes sont sincères, qu'ils reflètent bien la réalité des opérations, qu'ils portent sur tous les engagements pris par les gestionnaires locaux, tout en contribuant à améliorer la performance de la gestion locale. La compétence professionnelle des magistrats financiers, leur expérience, leur capacité d'expertise, leur rigueur morale, confèrent la qualité voulue au contrôle, qui justifie la confiance qui doit fonder les rapports entre les collectivités locales et les Chambres régionales des comptes. Celles-ci se gardent de toute instrumentalisation qui pourrait indisposer les gestionnaires locaux, notamment dans les périodes pré-électorales, et au contraire leur contrôle « permet de lever les réticences voire les suspicions qui pèsent parfois sur (eux) ». L'alternative à la pénalisation parfois excessive des acteurs locaux, réside dans un régime de responsabilité spécifique élaboré dans le cadre d'un droit public financier autonome et doté d'objectifs propres. Alors que les acteurs en charge de la gestion locale sont confrontés à un système devenu interactif et international complexe qui obère leur pouvoir de décision, la juridiction financière entend développer une culture de la responsabilité avec un régime modernisé. Celui-ci repose sur des règles de bonne gouvernance, au nombre desquelles se trouvent quelques grands principes composant une espèce de code de bonne conduite des gestionnaires des finances publiques. On y retrouve les notions d'éthique et de probité, de diligence, d'économie, d'efficacité et de performance, mais aussi de

111 J. CAILLOSSE, « Le droit administratif contre la performance ? », in A.J.D.A., p. 195 et s.

112 D. MIGAUD, « Il n'y a pas d'affaiblissement du contrôle des Chambres régionales des comptes», in AJDA, n 9/2012, p. 460.

113 H. M. CRUCIS, « L'article 47-2 de la constitution, la réforme de la Cour des comptes et la responsabilisation des gestionnaires de fonds publics », in AJDA., n 26/2009, p. 1410. 
vérification et de contrôle ${ }^{114}$. Toutes ces obligations professionnelles caractérisent la gestion publique moderne.

En définitive, le contrôle atteint son but lorsque les règles sont respectées, mais surtout lorsque les citoyens adhérent à la démocratie locale et si la qualité de vie des générations futures et du lien social est assurée. La transposition même amendée de la gestion d'entreprise aux collectivités locales, ne doit pas faire perdre de vue que ces dernières contribuent aussi à la construction et au fonctionnement de l'Etat. La gestion décentralisée et un certain esprit de compétition ne doivent pas nuire au devoir de solidarité. Il est clair que la qualité des institutions financières et celle des institutions politiques sont liées. Dans la mesure où la juridiction financière contribue au meilleur fonctionnement de la démocratie locale, le principe de responsabilité et d'évaluation doivent être au cœur de tout projet de décentralisation. La question de la responsabilité du système politique et administratif dans son ensemble, concerne aussi bien les collectivités locales que l'Etat.

\section{BIBLIOGRAPHIE SOMMAIRE COMPLÉMENTAIRE}

ABATE B., La nouvelle gestion publique, L.G.D.J., 2001

BARILARI A., "Quelques réflexions sur le contrôle des fonds publics », Revue du Trésor, $n^{\circ} 2 / 2003$, p. 83 et s.

BARILARI A. et BOUVIER M., La nouvelle gouvernance financière de l'Etat, L.G.D.J., 2004

BOUVIER M., «L'autonomie financière locale à travers les crises », Revue Française de Finances Publiques, $n^{\circ}$ 119/2012, p. 3 et $s$.

GIBERT P., «Le management public », Cahiers Français, juillet août 2004, n 321

HURON D. et SPINDLER J., Le management public local, L.G.D.J., 1998

LASCOMBE M., «La nouvelle gouvernance financière », Actualité Juridique de Droit Administratif, 2013, p. 228

LOMBARD M., « Le droit public économique face à la crise », Revue Française de Droit Administratif, 2010, p. 764

PICARD J.F., Finances locales, Lexis Nexis, 2013

PORTAL E., «La modernisation de la gestion des collectivités territoriales et l'évolution du droit financier local », Revue Française de Finances Publiques, n 81/2003, p. 224 et $\mathrm{s}$.

114 H.M.CRUCIS,op. cit., p. 1409. 
REVUE FRANCAISES DE FINANCES PUBLIQUES, Finances publiques : questions clés pour demain, ${ }^{\circ}$ 41/1993, Le Conseil d'Etat juge financier, n 70/2000-, Nouvelle gouvernance financière publique : grands enjeux de demain, $n^{\circ} 100 / 2007$, Fiscalité locale : quels enjeux ?, $\mathrm{n}^{\circ} 131 / 2015$

\section{KONTROLA NAD LOKALNIM UPRAVLJANJEM OD STRANE FINANCIJSKIH SUDOVA}

Svaki oblik decentralizacije podrazumijeva kontrolu nad lokalnim upravljanjem, kako bi se osigurao demokratski karakter, pravna pravilnost i gospodarska učinkovitost. Međutim, lokalno upravljanje ne možemo svesti samo na primjenu pravnih pravila niti pomiješati sa strogo gospodarskim pristupom. Zato je zadaća financijskog suda raznolika i posebna, a sastoji se od kombinacije upravnosudske kontrole nad lokalnim računima i kontrole koja nije upravnosudske naravi nad poštivanjem proračunskih pravila i nad učinkovitosti javne potrošnje kroz preispitivanje učinkovitosti lokalnog menadžmenta. Provjera zakonitosti i pravilnosti financijskih transakcija i opća zadaća u procjeni upravljanja, udružuju pravnu, gospodarsku i menadžersku logiku, u korist lokalne demokracije i uspješne i transparentne decentralizacije.

Ključne riječi: regionalni financijski sudovi, decentralizacija, javne financije, javno računovodstvo, proračunska kontrola, upravljanje, odgovornost, menadžment, lokalna demokracija 


\section{Prilog:}

INTERNA STRUKTURA REGIONALNOG FINANCIJSKOG SUDA Izvor : Michel BOUVIER, Les finances locales, ed. L.G.D.J., 2015, Stranica 255

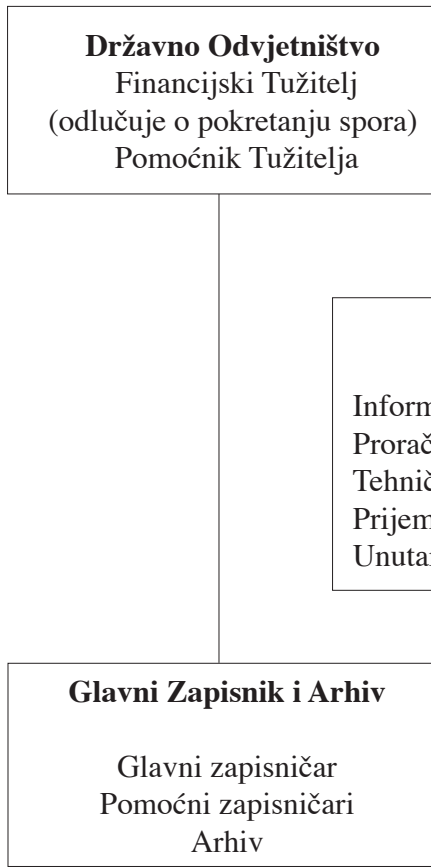

\section{Ured za Dokumentaciju}

Glavni sluzbenik za dokumentaciju / Pomoćnik Webmaster

Webmestre

\section{Opće Tajništvo}

Informativni Ured

Proračun - Knjigovodstvo

Tehnička služba

Prijemna služba

Unutarnja služba

\section{Predsjednik}

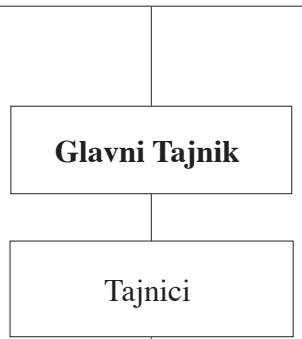

Tajnici

\section{ODJELI}

1.Odjel

Predsjednik Odjela

Suci

Asistenti sudaca za provjeravanje (financijskog djelovanja)

2. Odjel

Predsjednik Odjela

Suci

Asistenti za provjeravanje (financijskog djelovanja)

$N$. Odjel

Predsjednik Odjela / Suci/ Asistenti 